ITP-SB-98-23

EDINBURGH 98/3

\title{
Evolution of Color Exchange IN QCD HARD SCATTERING
}

\author{
NiKOLAOS KIDONAKIS \\ Department of Physics and Astronomy \\ University of Edinburgh, Edinburgh EH9 3JZ, Scotland, UK \\ Gianluca Oderda and George Sterman \\ Institute for Theoretical Physics \\ SUNY at Stony Brook, Stony Brook, NY 11794-3840, USA
}

\begin{abstract}
In QCD hard scattering cross sections, the color content of the underlying hard scattering evolves with a factorization scale. This evolution is controlled by an anomalous dimension matrix, specific to each hard-scattering reaction. Anomalous dimensions are determined from the renormalization of products of ordered exponentials of the gauge field, which describe the coherent radiation of gluons by incoming hadrons and the observed jets or particles of the final state. The anomalous dimensions depend on the kinematics of the underlying hard scattering, but are free of collinear singularities. A number of these matrices are available in the literature. Here, we exhibit one-loop mixing matrices for the full list of $2 \rightarrow 2$ reactions involving light quarks and gluons. The eight-byeight anomalous dimension matrix for gluon-gluon scattering shows a simplified structure in the basis corresponding to definite color exchange in the $t$-channel.
\end{abstract}




\section{Introduction}

Inclusive, short-distance hadronic cross sections may be factorized into convolutions of universal, nonperturbative parton distributions and/or fragmentation functions, and perturbative hard-scattering coefficient functions. In this procedure, a factorization scale $\mu$ separates nominally long- and short-distance components of the cross section. When $\mu$ is chosen large enough that the coupling $\alpha_{s}\left(\mu^{2}\right)$ is perturbative, it is possible to calculate the coefficient functions, and hence to determine the cross section, assuming that the relevant nonperturbative densities are known.

Factorization is an expression of quantum mechanical incoherence. The parton distributions summarize the internal dynamics of the incoming hadrons, and of their fragments that evolve into the final state. These distributions are independent of each other and of the fragmentation of scattered partons into jets and hadrons, in the final state. They are also incoherent with the short-distance dynamics that produces the hard scattering. Finally, they are incoherent with the dynamics of "noncollinear" gluons of any energy - from the hard scale $Q$ down to zero - that are radiated away from the incoming hadrons, or from the observed components of the final state [1, 2, 3].

Each of these components of a hard-scattering process: parton distributions, parton (or jet) fragmentation, hard scattering and soft gluons, may be thought of as governed by an effective field theory, in which the parton dynamics at differing scales and directions has been removed, and sometimes replaced by specific operators. At fixed order, coefficient functions combine contributions of quanta that are off-shell by the order of $Q$ with those from noncollinear soft gluons, because divergent soft-gluon behavior cancels, leaving finite contributions at each order in perturbation theory. These contributions, however, retain sensitivity to momentum scales much smaller than $\mu$, and may produce large, although finite, higher-order corrections.

Noncollinear soft-gluon dynamics is an important ingredient in a variety of resummed cross sections. Their first complete treatment was given by Collins and Soper for the transverse momentum $\left(Q_{T}\right)$ distribution of hadron pairs in $\mathrm{e}^{+} \mathrm{e}^{-}$annihilation [4. Because this cross section is not fully inclusive, it is necessary to resum (Sudakov) logarithms of $Q_{T}$ due to soft gluon emission to determine the cross section near $Q_{T}=0$, a procedure intensively studied for Drell-Yan pairs [0, 6. As is typical of such resummations, nonperturbative effects remain important phenomenologically at

realistic energy scales. In fact, the perturbative resummation suggests the functional form of these effects [6]. A similar resummation is necessary to define elastic scattering amplitudes for hadrons and partons at large momentum transfer and fixed angle [7, 8, 9, 10]. In both of these cases, leading logarithms are generated by soft gluons emitted collinear to incoming partons. Nevertheless, a treatment of noncollinear soft emission, i.e. soft gluons emitted at angles between the directions of the primary incoming and outgoing hard partons, is necessary to extend the formalism to nextto-leading logarithm and beyond. The pattern of this emission is essentially coherent [11]; as we shall see below, it is determined primarily by interference between emission from different hard partons. Despite this, it remains incoherent with the collinear dynamics of incoming and outgoing hard partons. That is, it factorizes from parton distributions and from suitably defined final-state jets. 
In inclusive quantities such as the Drell-Yan cross section $d \sigma / d Q^{2}$, soft gluon dynamics is somewhat hidden in higher order corrections to short-distance coefficient functions. It appears indirectly, through integrable logarithms at the "edge of phase space", where the observed final state uses up all partonic energy, leaving none over for QCD radiation. These phenomena have been studied in inclusive electroweak reactions such as the Drell-Yan cross section [12, 13], and more recently in inclusive QCD reactions such as heavy quark [14, 15, 16, 17, 18, 19, 20] and dijet production 21].

Beyond leading logarithms, all of these resummations require a treatment of soft, noncollinear, gluons. The hard scattering of partons of specific flavors involves, in general, a number of possible color exchanges. On quite general grounds, we expect this information to be reflected in observable properties of the final state [22]. At the same time, it is clear that if soft gluons are factorized from the hard scattering, the color content of the latter depends on the scale at which the separation is carried out. Nevertheless, physical cross sections cannot depend on this scale. The combination of factorization, and invariance under scale choice ensures the possibility of resummation in the scale dependence [23].

In this procedure, the emission of noncollinear soft gluons is described by matrix elements of products of ordered exponentials of the gluon field, coupled at a point representing the hard scattering [7, 17, 18, 20, 21]. The factorization scale between the hard and soft gluon functions may be interpreted as the renormalization scale for these operators. If the physical cross section is defined so that the soft gluon function depends on a single ratio of a phenomenological mass scale to the renormalization scale, then the entire soft function is determined by its anomalous dimensions.

Rather than review the specifics of QCD resummations in detail, we shall treat here one feature common to them all, the evolution of color exchange in noncollinear soft gluon emission. Our discussion will therefore treat gluons interacting with ordered exponentials instead of physical partons. We will present the full set of anomalous dimensions relevant to the scattering of light partons, including quark-quark, quarkantiquark (which have been presented previously), quark-gluon, and (by far the most complex case) gluon-gluon. Each of these partons will be replaced, for this discussion, by Wilson lines of the corresponding color representation. The justification for this approximation, and the detailed role that it plays in each cross section, may be found in the references cited above.

We begin, in Sec. 2, with a discussion of the renormalization of products of ordered exponentials, with the aim of isolating the evolution of color mixing. We shall see that, although the analysis immediately encounters collinear divergences, it is easy to identify finite anomalous dimensions that govern the mixing of color. We go on, in Sec. 3, to recall, for definiteness, the soft gluon contribution to inclusive jet cross sections in hadronic scattering [21]. This "soft function" is manifestly free of collinear singularities, and its evolution, with mixing, reflects the color content of the underlying hard scattering. In Sec. 4, we introduce a color basis notation that is convenient for the solution of the evolution equation for the soft function. This is followed, in Secs. 5 and 6, by the computation of the anomalous dimension matrices for various processes, along with their eigenvalues and eigenvectors. We have included 
two appendices that describe some of the details of the calculations.

\section{Soft Anomalous Dimensions from Wilson Lines}

Our discussion begins with ordered exponentials, or Wilson lines. A general Wilson line is labelled by a path in space-time, $C$, beginning at point $z$ and ending at point $z^{\prime}$

$$
W\left[C ; z^{\prime}, z\right]=P \exp \left[-i g \int_{\lambda_{1}}^{\lambda_{2}} d \eta \frac{d y(\lambda)}{d \eta} \cdot A(y(\eta))\right] .
$$

The variable of integration $\eta$ parameterizes $C$, with $y\left(\lambda_{1}\right)=z, y\left(\lambda_{2}\right)=z^{\prime}$.

Wilson lines have myriad applications in quantum field theory, but their interest here is as a model for the radiation of soft gluons by fast-moving partons (quarks and gluons). Any hard-scattering event, be it based on electroweak or QCD hard scattering, results in nonabelian radiation, in much the same manner as in classical electrodynamics. Indeed, as in classical electrodynamics, the pattern of soft radiation is determined by the charge currents at long times before and after the scattering event. The factorization properties of inclusive hard-scattering cross sections in QCD rely on this feature [3].

Initial and final-state partons are represented by ordered exponentials in which $C$ is a straight line, in the direction of the four-velocity, $\beta$, of the relevant parton. For inclusive cross sections, the lines extend from a common point $x$ to infinity, either from the distant past, or toward the distant future. We shall adopt the notation of Ref. 21] for such Wilson lines,

$$
\Phi_{\beta}^{(f)}\left(\lambda_{2}, \lambda_{1} ; x\right)=P \exp \left(-i g \int_{\lambda_{1}}^{\lambda_{2}} d \eta \beta \cdot A^{(f)}(\eta \beta+x)\right),
$$

where the gauge field $A^{(f)}$ is a matrix in the representation of the flavor $f$. Comparing this definition with Eq. (11), we have $y(\eta)=\eta \beta+x$.

Although the Wilson lines $\Phi$ in Eq. (2) extend to infinity, they reproduce the dynamics of the gauge field for only a finite time before and after a hard scattering. At long times, nonperturbative dynamics dominate, nonabelian charges are neutralized, and a picture in terms of isolated currents fails utterly. Therefore, the procedure applies only to cross sections for which such long-distance effects cancel in perturbation theory. The final answers for such quantities are insensitive to the infrared properties of amplitudes generated from Wilson lines of the form of Eq. (2), although it may be necessary to deal with these properties at intermediate steps in any calculation. In fact, it is their ultraviolet behavior that we shall find most interesting.

This approach has been applied to the classic electroweak hard scattering processes, $\mathrm{e}^{+} \mathrm{e}^{-}$annihilation, DIS and Drell-Yan vector boson production [12, 13, 24]. In each of these cases, the relevant operator is a product of two $\Phi$ 's coupled at a color singlet vertex. Equivalently it is a single Wilson line, whose direction changes discontinuously at a single point. For $\mathrm{e}^{+} \mathrm{e}^{-}$annihilation, the curve $C$ consists of two straight paths, both extending to positive infinity in time, describing the creation of 
a quark pair at a point. For DIS, $C$ comes in from negative infinity and changes direction, describing the scattering of a quark or antiquark, while for Drell-Yan, $C$ represents the annihilation of a pair.

For definiteness, we consider the Wilson line analog of the Drell-Yan cross section [24], where the configuration just described corresponds to the product

$$
W_{b_{2}, b_{1}}^{(\mathrm{DY})}(x)=\delta_{a_{1} a_{2}} \Phi_{\beta_{2}}^{(\bar{q})}(0,-\infty ; x)_{a_{2} b_{2}} \Phi_{\beta_{1}}^{(q)}(0,-\infty ; x)_{a_{1} b_{1}} .
$$

The vertex at $x$ is associated with ultraviolet divergences, as are self-energies of the eikonal lines. The divergent one-loop diagrams are shown in Fig. 1. These vertex and self-energy diagrams are conveniently renormalized by perturbative counterterms, corresponding to multiplicative renormalization of the composite operators [25, 26, 27,

$$
W^{(\mathrm{DY})(0)}(x)=Z_{W}^{(\mathrm{DY})}\left(\alpha_{s}, \frac{\beta_{1} \cdot \beta_{2}}{\sqrt{\beta_{1}^{2} \beta_{2}^{2}}}\right) W^{(\mathrm{DY})}(x),
$$

where $W^{(\mathrm{DY})(0)}$ is the bare operator, and where we have used the invariance of $W$ under rescalings of the velocities $\beta_{i}$. In the following, we suppress dependence on $x$, the position of the composite vertex. The independence of the unrenormalized operator from the renormalization scale $\mu$ implies that the renormalized operator satisfies the renormalization group ( $R G$ ) equation!,

$$
\mu \frac{d}{d \mu} W^{(\mathrm{DY})}=\Gamma_{W}\left(\alpha_{s}, \frac{\beta_{1} \cdot \beta_{2}}{\sqrt{\beta_{1}^{2} \beta_{2}^{2}}}\right) W^{(\mathrm{DY})} .
$$

The anomalous dimension $\Gamma_{W}\left(\alpha_{s}, \beta_{i}\right)$ is the same for all color singlet products of the form of Eq. (3), and is sometimes referred to as $\Gamma_{\text {cusp }}$ [27.

Because $W^{(\mathrm{DY})}\left(\alpha_{s}, \beta_{i}, n\right)$ is a gauge-invariant operator, so is $\Gamma_{W}$. A direct calculation verifies that, as suggested by Eq. (5), $\Gamma_{W}$ is somewhat unusual for an anomalous dimension, in that it diverges in the limit of light-like velocities for the external lines, $\beta_{i}^{2}=0$. Fixing the $\beta_{i}^{2}$ to be small, but nonvanishing, we find the explicit expression

$$
\Gamma_{W}=\frac{\alpha_{s}}{\pi} C_{F}\left[\ln \left(\frac{-2 \beta_{1} \cdot \beta_{2}}{\sqrt{\beta_{1}^{2} \beta_{2}^{2}}}\right)-1\right] .
$$

The divergence at light-like velocities is a collinear enhancement, due to the emission of gluons in the direction of the eikonal line. In suitably-defined inclusive cross sections, the corresponding collinear divergences either cancel, or are absorbed into parton distributions [3]. We shall see that the same is true for a product of two Wilson lines like Eq. (3) and its generalizations. Specifically, the collinear divergences in

\footnotetext{
${ }^{1}$ Strictly speaking, the multiplicative renormalization in Eq. (4), and the corresponding RG equation (5) apply to the renormalization of the composite operator only; the renormalization associated with the QCD Lagrangian is assumed to have been carried out. In [25] the renormalization proof was formulated in terms of the vacuum expectation value of the corresponding loop.
} 
$\Gamma_{W}$ are universal, and may be factored from the underlying local coupling. In computing loop corrections to $W$ and $\Phi$, we treat the eikonal lines as on-shell particles, normalized by the square root of the residue of the eikonal two-point function. This ensures gauge invariance.

To demonstrate factorizability, we shall find it useful to work in an axial gauge, $n \cdot A=0$, with $n \cdot \beta_{i} \neq 0, i=1,2$. In any gauge, a single Wilson line $\Phi_{\beta}^{(q)}$ in quark representation, defined on a ray in the direction of velocity $\beta$ is also multiplicatively renormalizable [25],

$$
\Phi_{\beta}^{(q)(0)}=Z_{\Phi}\left(\alpha_{s}, \frac{|\beta \cdot n|}{\sqrt{\beta^{2}\left|n^{2}\right|}}\right) \Phi_{\beta}^{(q)},
$$

where again, rescaling invariance in the velocity and the gauge vector limits possible arguments of $Z$. Eq. (可) implies that $\Phi_{\beta}^{(q)}$ satisfies a renormalization group equation

$$
\mu \frac{d}{d \mu} \Phi_{\beta}^{(q)}=\Gamma_{\Phi}^{(q)}\left(\alpha_{s}, \frac{|\beta \cdot n|}{\sqrt{\beta^{2}\left|n^{2}\right|}}\right) \Phi_{\beta}^{(q)},
$$

with $\Gamma_{\Phi}^{(q)}$ another anomalous dimension. Clearly, $\Phi^{(\bar{q})}$ requires the same renormalization constant.

The one-loop expression for $\Gamma_{\Phi}$ in axial gauges has the same collinear $\left(\beta^{2} \rightarrow 0\right)$ divergence as $\Gamma_{W}$, but now through gauge-dependent logarithms,

$$
\Gamma_{\Phi}^{(q)}\left(\alpha_{s}, \frac{|\beta \cdot n|}{\sqrt{\beta^{2}\left|n^{2}\right|}}\right)=\frac{\alpha_{s}}{\pi} C_{F}\left[\ln \left(\frac{2|\beta \cdot n|}{\sqrt{\beta^{2}\left|n^{2}\right|}}\right)-1\right] .
$$

Evidently, the collinear divergences in this anomalous dimension exactly match those of $\Gamma_{W}^{(\mathrm{DY})}$, with the opposite sign. As we shall see, this result is quite general.

To make use of this correspondence, let us define a new operator, which generates the sum of diagrams, containing the Drell-Yan vertex, that cannot be reduced by cutting a single eikonal line. We do this by simply dividing out the vacuum expectation values of the two external Wilson lines in Eq. (3), which defines $W^{(\mathrm{DY})}$,

$$
S^{(\mathrm{DY})}=W^{(\mathrm{DY})}\left\langle 0\left|\Phi_{\beta_{1}}^{(q)}(0,-\infty ; 0)\right| 0\right\rangle^{-1}\left\langle 0\left|\Phi_{\beta_{2}}^{(\bar{q})}(0,-\infty ; 0)\right| 0\right\rangle^{-1},
$$

where in the vacuum expectation value a trace and an average of color of the operator $\Phi$ is assumed.

Given the RG equations for $W^{(\mathrm{DY})}$ and the $\Phi$ 's, the function $S^{(\mathrm{DY})}$ satisfies a renormalization group equation of exactly the same sort as $W^{(\mathrm{DY})}$,

$$
\mu \frac{d}{d \mu} S^{(\mathrm{DY})}=\Gamma_{S}\left(\alpha_{s}, \beta_{i}, n\right) S^{(\mathrm{DY})},
$$

with a "soft" anomalous dimension,

$$
\begin{aligned}
\Gamma_{S} & =\Gamma_{W}-\Gamma_{\Phi}^{(q)}\left(\alpha_{s}, \frac{\left|\beta_{1} \cdot n\right|}{\sqrt{\beta_{1}^{2}\left|n^{2}\right|}}\right)-\Gamma_{\Phi}^{(\bar{q})}\left(\alpha_{s}, \frac{\left|\beta_{2} \cdot n\right|}{\sqrt{\beta_{2}^{2}\left|n^{2}\right|}}\right) \\
& =\frac{\alpha_{s}}{\pi} C_{F}\left[1+\ln \left(\frac{-\beta_{1} \cdot \beta_{2}\left|n^{2}\right|}{2\left|\beta_{1} \cdot n\right|\left|\beta_{2} \cdot n\right|}\right)\right] .
\end{aligned}
$$


We may think of $\Gamma_{S}$ as the "coherent" part of the "cusp" anomalous dimension $\Gamma_{W}$, that is, the part that depends on the specific color-singlet vertex in Eq. (3), but is independent of the mutually incoherent collinear behavior of the lines themselves. Our definition of $\Gamma_{S}$ is, however, somewhat arbitrary, and we might change it through a finite renormalization and/or a change of gauge. A natural choice is $A^{0}=0$ gauge in a frame where $\beta_{1}^{\mu}=\delta_{\mu+}$ and $\beta_{2}^{\mu}=\delta_{\mu-}$, in which case $\Gamma_{S}=\left(\alpha_{s} / \pi\right) C_{F}(1-i \pi)$ [12], but we are even free to make $\Gamma_{S}^{(\mathrm{DY})}$ vanish. Any choice leads to the same amplitude.

The value of this approach is found by generalizing $W^{(\mathrm{DY})}$ to products of multiple Wilson lines. For QCD hard-scattering processes, products of four lines are most interesting, and illustrate the general case. As noted above, the collinear divergences represented by Eq. (6) are more general than the color-singlet vertex in $W^{(D Y)}$, precisely because they may be factored from this vertex, and from each other. In the anomalous dimensions, which appear eventually in exponents, this factorization is guaranteed to be additive.

In the context of QCD hard-scattering [17, 18, 19, 20, 21], most of the above considerations apply, although now with a more complex and interesting color flow. In this paper, we shall be concerned specifically with soft radiation for cross sections due to $2 \rightarrow 2$ scattering of light quarks and gluons at short distances, of relevance to high- $p_{T}$ jet production. Analogous reactions are also at the basis of heavy quark production. In this larger context, the value of isolating single-logarithmic soft gluon dynamics from corresponding, universal, collinear dynamics is clear. We shall review very briefly in Sec. 3 the role of products of Wilson lines in hard cross sections, using as an example the resummation of threshold corrections in jet cross sections. First, however, we show how the generalization of the color-singlet product $W^{(\mathrm{DY})}$ leads naturally to a matrix of anomalous dimensions that describe the mixing of color with changing scales.

Following Ref. [21], we introduce a class of operators coupling four Wilson lines, corresponding to the primary partons involved in a hard scattering,

$$
\begin{aligned}
W_{I}^{(\mathrm{f})}(x)_{\left\{r_{k}\right\}}= & \sum_{\left\{d_{i}\right\}} \Phi_{\beta_{2}}^{\left(f_{2}\right)}(\infty, 0 ; x)_{r_{2}, d_{2}} \Phi_{\beta_{1}}^{\left(f_{1}\right)}(\infty, 0 ; x)_{r_{1}, d_{1}} \\
& \times\left(c_{I}^{(\mathrm{f})}\right)_{d_{2} d_{1}, d_{B} d_{A}} \Phi_{\beta_{A}}^{\left(f_{A}\right)}(0,-\infty ; x)_{d_{A}, r_{A}} \Phi_{\beta_{B}}^{\left(f_{B}\right)}(0,-\infty ; x)_{d_{B}, r_{B}},
\end{aligned}
$$

and a corresponding irreducible soft function,

$$
S_{I}^{(\mathrm{f})}=W_{I}^{(\mathrm{f})} \prod_{i=A, B}\left\langle 0\left|\Phi_{\beta_{i}}^{\left(f_{i}\right)}(0,-\infty ; 0)\right| 0\right\rangle^{-1} \prod_{i=1,2}\left\langle 0\left|\Phi_{\beta_{i}}^{\left(f_{i}\right)}(\infty, 0 ; 0)\right| 0\right\rangle^{-1} .
$$

As above, $\beta_{i}$ represents the four-velocities of initial- and final-state partons. The superscript $\mathrm{f}$ represents a scattering process,

$$
f_{A}\left(\ell_{A}, r_{A}\right)+f_{B}\left(\ell_{B}, r_{B}\right) \rightarrow f_{1}\left(p_{1}, r_{1}\right)+f_{2}\left(p_{2}, r_{2}\right)
$$

involving four partons, with flavors $f_{i}$, incoming momenta $l_{i}$ and outgoing momenta $p_{i}$, and color $r_{i}$. The color tensor $\left(c_{I}^{(\mathrm{f})}\right)_{d_{2} d_{1}, d_{B} d_{A}}$ describes the couplings of the ordered 
exponentials with each other in color space. If, for example, the incoming lines are quark and antiquark, the indices $r_{i}, d_{j}$ are in the fundamental representations of $S U(3)$. Examples of bases for the color tensors $c_{I}$ then include: singlet and octet matrices in the $s$ - or in the $t$-channel, or $s$-channel color singlet and $t$-channel color singlet.

The relationship between the $W_{I}$ and the $S_{I}$ is shown in Fig. 2 in axial gauges. The left-hand side of the figure shows a general momentum configuration of a "leading region" [3], that contributes logarithmic behavior to (the vacuum expectation value of) $W_{I}^{(\mathrm{f})}$; lines have been grouped according to their momentum flow. Such configurations may be identified by standard Minkowski-space power counting [3]. As indicated in the diagram, all lines fall either into "jet-like" subdiagrams, labelled 1, 2, $A, B$, or into a "soft" subdiagram, labelled $S$. The jet subdiagrams consist of lines that are all on-shell with light-like momenta parallel to the directions of the corresponding $\beta_{i}$. The momenta of lines in $S$ vanish in all four components. As indicated in the figure, in each such "leading" region, all collinear contributions factor into the self energies, in axial gauges. Demonstrations of this factorization, based on Ward identities in QCD may be found in Refs. [4], [1] and [3] in the case of jets of partons. The eikonal lines in Fig. 2 obey the same Ward identities as do quark lines, and do not change these arguments.

Under renormalization, the set of operators $W_{I}^{(\mathrm{f})}$, and hence the $S_{I}^{(\mathrm{f})}$, will mix in general. Because the eikonal lines do not include recoil effects, they cannot annihilate, and there is no mixing between vertices that link other sets of Wilson lines (corresponding to other flavor flows f). The corresponding renormalization group equation is now in terms of a matrix of anomalous dimensions,

$$
\begin{aligned}
\mu \frac{d}{d \mu} W_{I}^{(\mathrm{f})} & =\left(\Gamma_{W}^{(\mathrm{f})}\right)_{J I}\left(\alpha_{s}, \beta_{i}, \beta_{j}\right) W_{J}^{(\mathrm{f})} \\
\mu \frac{d}{d \mu} S_{I}^{(\mathrm{f})} & =\left(\Gamma_{S}^{(\mathrm{f})}\right)_{J I}\left(\alpha_{s}, \frac{\beta_{i} \cdot \beta_{j}\left|n^{2}\right|}{\left|\beta_{i} \cdot n\right|\left|\beta_{j} \cdot n\right|}\right) S_{J}^{(\mathrm{f})}
\end{aligned}
$$

where in the arguments of $\Gamma_{S}, i \neq j$. The two anomalous dimension matrices are related by a generalization of Eq. (12) for Drell-Yan,

$$
\Gamma_{S, I J}^{(\mathrm{f})}\left(\alpha_{s}, \beta_{i}, n\right)=\Gamma_{W, I J}^{(\mathrm{f})}\left(\alpha_{s}, \beta_{i}, \beta_{j}\right)-\delta_{I J} \sum_{i=1}^{4} \Gamma_{\Phi}^{\left(f_{i}\right)}\left(\alpha_{s}, \frac{\left|\beta_{i} \cdot n\right|}{\sqrt{\beta_{i}^{2}\left|n^{2}\right|}}\right)
$$

The anomalous dimensions for $W_{I}^{(\mathrm{f})}$ include collinear divergences in the limit of vanishing $\beta_{i}^{2}$. These divergences, however, are universal, and may be absorbed into the renormalization of the $\Phi$ 's. This is possible precisely because any collinear divergence may be absorbed into a self-interaction of one of the Wilson lines, as argued above. Since the renormalization of self-energy graphs does not mix colors at the vertex, collinear divergences in $\Gamma_{W}^{(\mathrm{f})}$, Eq. (16), can only appear times the identity in the space of color tensors. Therefore the soft anomalous dimension matrix $\Gamma_{S, I J}^{(\mathrm{f})}$ summarizes the mixing of operators under renormalization, but is free from collinear divergences, as long as $\beta_{i} \cdot \beta_{j} \neq 0$ for $i \neq j$. The soft anomalous dimension $\Gamma_{S}^{(\mathrm{f})}$ will be, as in the 
case of $\Gamma_{S}^{(\mathrm{DY})}$ above, free of universal collinear divergences, not only at one loop, but to all orders in perturbation theory.

Note that the choice of finite renormalization for the $\Phi$ 's, and of overall gauge, encountered above, may be interpreted as a renormalization scheme for defining the composite vertex and its soft anomalous dimension. In the next section, we shall exhibit a class of infrared safe soft functions that occur in the threshold resummation

of dijet cross sections [21], and whose evolution is controlled by the tensors $\Gamma_{S, I J}^{(\mathrm{f})}$.

\section{Soft Functions in Dijet Production}

In this section we recall the role that eikonal operators like those discussed above play in the resummation of threshold corrections in dijet production,

$$
h_{A}\left(p_{A}\right)+h_{B}\left(p_{B}\right) \longrightarrow J_{1}\left(p_{1}\right)+J_{2}\left(p_{2}\right)+X(k),
$$

at fixed dijet invariant mass,

$$
M_{J J}^{2}=\left(p_{1}+p_{2}\right)^{2}
$$

and at fixed rapidity difference,

$$
\Delta y=\frac{1}{2} \ln \left(\frac{p_{1}^{+} p_{2}^{-}}{p_{1}^{-} p_{2}^{+}}\right)=\ln \left(\frac{u}{t}\right)
$$

with $t$ and $u$ the usual Mandelstam variables, defined as

$$
\begin{aligned}
t & =\left(p_{A}-p_{1}\right)^{2} \\
u & =\left(p_{A}-p_{2}\right)^{2} .
\end{aligned}
$$

As discussed in Ref. [21], many other choices of cross section involve essentially the same physics. Again to be specific, we assume that the two final-state jets in (18) are identified by cones of opening angles $\delta_{i}$.

We shall be concerned with the contribution to this cross section from an underlying partonic scattering like Eq. (15). In particular, we shall review some relevant features of the resummation, in this cross section, of "threshold" corrections to the hardscattering function. These are corrections of the general form $\left[\ln ^{n}(1-z) /(1-z)\right]_{+}$, with $z=M_{J J}^{2} / \hat{s}$, where $\hat{s}$ is the invariant mass squared of the partons that initiate the process. Referring to Eq. (15), we take $\ell_{A}=x_{A} p_{A}, \ell_{B}=x_{B} p_{B}$, with $p_{A}$ and $p_{B}$ the momenta of the incoming hadrons and $x_{A, B}$ momentum fractions, so that $\hat{s}=2 x_{A} x_{B} p_{A} \cdot p_{B}$. We represent the coupling of soft gluons to the hard partons of the flavors $f_{A}, f_{B}, f_{1}$ and $f_{2}$ by Wilson lines, as in Eq. (2), tied together at the vertices of Eq. (13). In terms of the operators $W_{I}^{(\mathrm{f})}$ we define a dimensionless "eikonal cross section", describing the emission of gluons by hard partons in eikonal approximation,

$$
\begin{aligned}
\sigma_{L I}^{(\mathrm{f}, \mathrm{eik})}\left(\frac{w M_{J J}}{\mu}, \Delta y, \alpha_{s}\left(\mu^{2}\right), \delta_{i}, \epsilon\right) & =\sum_{\xi} \delta\left(w-w^{(\mathrm{eik})}\left(\xi, \delta_{i}\right)\right) \\
& \times\left\langle 0\left|\bar{T}\left[\left(W_{L}^{(\mathrm{f})}(0)\right)_{\left\{b_{i}\right\}}^{\dagger}\right]\right| \xi\right\rangle\left\langle\xi\left|T\left[W_{I}^{(\mathrm{f})}(0)_{\left\{b_{i}\right\}}\right]\right| 0\right\rangle,
\end{aligned}
$$


where $\xi$ designates a set of intermediate states. The eikonal cross section depends on the rapidity difference $\Delta y$ (or equivalently, the partonic center-of-mass scattering angle) through the relative directions of the Wilson lines coupled at each operator $W^{(\mathrm{f})}$. We choose the dimensionless weight $w$ in Eq. (22) to reproduce the phase space available to soft gluons near partonic threshold $(z=1)$ [21]. For the cross section in question, this requires that we fix the total energy emitted by the eikonal lines into the final state, but outside the cones $\delta_{i}$. Denoting this energy as $k_{\xi}^{0}$, we have

$$
w^{(\mathrm{eik})}\left(\xi, \delta_{i}\right)=\frac{2 k_{\xi}^{0}}{M_{J J}}
$$

To handle the collinear divergences of $\sigma^{(\mathrm{f}, \text { eik })}$ due to initial state interactions, and to avoid infinite energy emitted into the cones, we may think of the eikonals as having small "mass", $\beta_{i}^{2}$. We shall not need this regulator in any of our explicit calculations below, however.

As in the previous section, we want to isolate the contributions of noncollinear gluons, which control color mixing. For this purpose we factorize the eikonal cross section, Eq. (22), in the same manner as a hadronic cross section [3, 21]. Initial-state collinear divergences can be factorized into eikonal "parton distributions", which we label $j_{\text {IN }}$. Analogously, final-state collinear enhancements may be factorized into outgoing "jet" functions, jout.

The result of this procedure is to write the eikonal cross section as a convolution of an infrared safe soft function $S_{L I}^{(\mathrm{f})}$ with the functions $j_{\mathrm{IN}}$ and $j_{\mathrm{OUT}}$,

$$
\begin{aligned}
& \sigma_{L I}^{(\mathrm{f}, \mathrm{eik})}\left(\frac{w M_{J J}}{\mu},\right.\left.\Delta y, \alpha_{s}\left(\mu^{2}\right), \delta_{i}, \epsilon\right)= \\
& \int_{0}^{1} d w_{A}^{\prime} d w_{B}^{\prime} d w_{1}^{\prime} d w_{2}^{\prime} d w_{S}^{\prime} \delta\left(w-w_{1}^{\prime}-w_{2}^{\prime}-w_{A}^{\prime}-w_{B}^{\prime}-w_{S}^{\prime}\right) \\
& \quad \times \prod_{c=A, B} j_{\mathrm{IN}}^{\left(f_{c}\right)}\left(\frac{w_{c}^{\prime} M_{J J}}{\mu}, \alpha_{s}\left(\mu^{2}\right), \epsilon\right) \prod_{d=1,2} j_{\mathrm{OUT}}^{\left(f_{d}\right)}\left(\frac{w_{d}^{\prime} M_{J J}}{\mu}, \alpha_{s}\left(\mu^{2}\right), \delta_{d}\right) \\
& \quad \times S_{L I}^{(\mathrm{f})}\left(\frac{w_{S}^{\prime} M_{J J}}{\mu}, \Delta y, \alpha_{s}\left(\mu^{2}\right)\right) .
\end{aligned}
$$

Here $w_{i}^{\prime}=2 k_{i}^{\prime 0} / M_{J J}$, with $k_{i}^{\prime 0}$ the energy of partons emitted outside the cones, and associated with the function labelled by $i=1,2, A, B, S$. We are using the incoherence of these "effective theories", as mentioned in the introduction.

For completeness, we give definitions for the incoming eikonal jets [17, 21]:

$$
\begin{aligned}
& j_{\mathrm{IN}}^{\left(f_{i}\right)}\left(\frac{w_{i}^{\prime} M_{J J}}{\mu}, \alpha_{s}\left(\mu^{2}\right), \epsilon\right)=\frac{M_{J J}}{2 \pi} \int_{-\infty}^{\infty} d y_{0} \mathrm{e}^{-i w_{i}^{\prime} M_{J J} y_{0}} \\
& \times\left\langle 0\left|\operatorname{Tr}\left\{\bar{T}\left[\Phi_{\beta_{i}}^{\left(f_{i}\right) \dagger}(0,-\infty ; y)\right] T\left[\Phi_{\beta_{i}}^{\left(f_{i}\right)}(0,-\infty ; 0)\right]\right\}\right| 0\right\rangle,
\end{aligned}
$$

with $i=A, B$ and $y^{\nu}=\left(y_{0}, \overrightarrow{0}\right)$ a vector at the spatial origin. The argument $\epsilon=4-D$, in $D$ dimensions, denotes the presence of collinear singularities in $j_{\mathrm{IN}}^{\left(f_{i}\right)}$. Similarly, for 
the outgoing eikonal jets we have [21],

$$
\begin{aligned}
& j_{\mathrm{OUT}}^{\left(f_{i}\right)}\left(\frac{w_{i}^{\prime} M_{J J}}{\mu}, \alpha_{s}\left(\mu^{2}\right), \delta_{i}\right)=\sum_{\xi} \delta\left(w_{i}^{\prime}-w_{i}^{(\mathrm{eik})}\left(\xi, \delta_{i}\right)\right) \\
& \times\left\langle 0\left|\operatorname{Tr}\left\{\bar{T}\left[\Phi_{\beta_{i}}^{\left(f_{i}\right) \dagger}(\infty, 0 ; 0)\right]|\xi\rangle\langle\xi| T\left[\Phi_{\beta_{i}}^{\left(f_{i}\right)}(\infty, 0 ; 0)\right]\right\}\right| 0\right\rangle,
\end{aligned}
$$

with $i=1,2$. In this case, collinear singularities cancel due to the jet definition in the sum over final states, and the infrared regulator $\epsilon$ is replaced by the cone size $\delta_{i}$.

Mellin transforms of the eikonal cross section with respect to the weight conveniently isolate contributions from noncollinear soft gluons that are singular at partonic threshold [21. If we take the Mellin transform of Eq. (24),

$$
\int_{0}^{1} d w(1-w)^{N-1} \sigma_{L I}^{(\mathrm{f}, \mathrm{eik})}\left(\frac{w M_{J J}}{\mu}, \Delta y, \alpha_{s}\left(\mu^{2}\right), \delta_{i}, \epsilon\right),
$$

the convolution in the eikonal weights becomes a product of moments, up to corrections that vanish as a power of the moment variable $N$ for large $N$. Hence the moments of the soft function can be extracted from the eikonal cross section, as follows,

$$
\begin{aligned}
\tilde{S}_{L I}^{(\mathrm{f})}\left(\frac{M_{J J}}{N \mu}, \Delta y, \alpha_{s}\left(\mu^{2}\right)\right)= & \frac{\tilde{\sigma}_{L I}^{(\mathrm{f}, \mathrm{eik})}\left(\frac{M_{J J}}{N \mu}, \Delta y, \alpha_{s}\left(\mu^{2}\right), \delta_{i}, \epsilon\right)}{\tilde{j}_{\mathrm{IN}}^{\left(f_{A}\right)}\left(\frac{M_{J J}}{N \mu}, \alpha_{s}\left(\mu^{2}\right), \epsilon\right) \tilde{j}_{\mathrm{IN}}^{\left(f_{B}\right)}\left(\frac{M_{J J}}{N \mu}, \alpha_{s}\left(\mu^{2}\right), \epsilon\right)} \\
& \times \frac{1}{\tilde{j}_{\mathrm{OUT}}^{\left(f_{1}\right)}\left(\frac{M_{J J}}{N \mu}, \alpha_{s}\left(\mu^{2}\right), \delta_{1}\right) \tilde{j}_{\mathrm{OUT}}^{\left(f_{2}\right)}\left(\frac{M_{J J}}{N \mu}, \alpha_{s}\left(\mu^{2}\right), \delta_{2}\right)} .
\end{aligned}
$$

All the leading power singularities, $\left[\frac{\ln ^{m}(1-z)}{1-z}\right]_{+}$, may be reconstructed from logarithms of the leading power $\left(\mathcal{O}\left(N^{0}\right)\right) N$ dependence.

We recognize Eq. (28) as a generalization of Eq. (14) to the moments of the cross section. By removing the mutually-incoherent jet factors, which in axial gauge correspond simply to cut parton self-energies, we isolate in the function $S_{L I}^{(\mathrm{f})}$ the interference of amplitudes in which gluons are emitted by one eikonal line and absorbed by another. In axial gauge, cut diagrams of this topology incorporate what we might describe as "interjet" coherent [11] radiation in the cross section.

We have not yet discussed the renormalization of the soft function and eikonal jets. In the next section we will see that renormalization leads to the exponentiation of the $N$-dependence of the soft function, and hence of the energy dependence of its transform.

Now let us relate the soft function $\tilde{S}_{L I}$ to threshold resummation for dijet cross sections. To derive singular behavior at partonic threshold, we consider moments with respect to $\tau=M_{J J}^{2} / S$ for perturbative, partonic cross sections, in which the external hadrons are replaced by partons in an infrared-regulated theory. In Ref. 
[21], we showed that, up to next-to-leading logarithms, this cross section factorizes under such a Mellin transform,

$$
\begin{aligned}
\int_{0}^{1} d \tau \tau^{N-1} S^{2} \frac{d \sigma_{f_{A} f_{B} \rightarrow J_{1} J_{2}}\left(S, \delta_{1}, \delta_{2}\right)}{d M_{J J}^{2} d \Delta y}=\sum_{\mathrm{f}} \sum_{I L} H_{I L}^{(\mathrm{f})}\left(\frac{M_{J J}}{\mu}, \Delta y, \alpha_{s}\left(\mu^{2}\right)\right) \\
\times \tilde{\psi}_{f_{A} / f_{A}}\left(N, \frac{M_{J J}}{\mu}, \alpha_{s}\left(\mu^{2}\right), \epsilon\right) \tilde{\psi}_{f_{B} / f_{B}}\left(N, \frac{M_{J J}}{\mu}, \alpha_{s}\left(\mu^{2}\right), \epsilon\right) \\
\times \tilde{S}_{L I}^{(\mathrm{f})}\left(\frac{M_{J J}}{\mu N}, \Delta y, \alpha_{s}\left(\mu^{2}\right)\right) \\
\times \tilde{J}^{\left(f_{1}\right)}\left(N, \frac{M_{J J}}{\mu}, \alpha_{s}\left(\mu^{2}\right), \delta_{1}\right) \tilde{J}^{\left(f_{2}\right)}\left(N, \frac{M_{J J}}{\mu}, \alpha_{s}\left(\mu^{2}\right), \delta_{2}\right) .
\end{aligned}
$$

The factorized cross section is illustrated in cut diagram notation in Fig. 3. Here, $S_{L I}$ is exactly the matrix of soft eikonal functions constructed above. $H_{I L}$ absorbs quanta, represented by $h_{L}^{*}$ and $h_{I}$ (see also Eq. (31) below), which are off-shell by the order of $M_{J J}$. The remaining functions $\psi$ and $J$ include, respectively, virtual and final-state partons parallel to the incoming hadrons (partons) and to the observed outgoing jets. Explicit definitions of the jet functions are given in Ref. [21]. Their detailed features are not of interest here, primarily because they are color diagonal, and incoherent with the soft radiation included in $S_{L I}$. Our interest is in the interplay of color structures between the soft tensor $S_{L I}$ and the hard color tensor $H_{I L}$.

\section{Color Evolution in Jet Cross Sections}

In this section, we exhibit the evolution of color exchange in hard scattering. Although our notation is taken from the dijet cross section of the previous section, it is quite general.

\subsection{Evolution for Soft and Hard Functions}

The composite operators of the soft function $\tilde{S}_{L I}^{(\mathrm{f})}$, as we saw before, require renormalization. The matrices $H$ and $S$ occur in a product in Eq. (29) and (consistent with the discussion of Sec. 2), must therefore renormalize multipicatively, with separate renormalization factors for the amplitude and the complex conjugate [7, 23],

$$
\begin{aligned}
H_{I L}^{(\mathrm{f})} \stackrel{(0)}{I L} & =\prod_{i=A, B, 1,2} Z_{i}^{-1}\left(Z_{S}^{(\mathrm{f})-1}\right)_{I C} H_{C D}^{(\mathrm{f})}\left[\left(Z_{S}^{(\mathrm{f}) \dagger}\right)^{-1}\right]_{D L} \\
S_{L I}^{(\mathrm{f})} & =\left(Z_{S}^{(\mathrm{f}) \dagger}\right)_{L B} S_{B A}^{(\mathrm{f})} Z_{S, A I}^{(\mathrm{f})} .
\end{aligned}
$$

Here $Z_{i}$ is the wavefunction renormalization constant of the $i$ th incoming parton field (of flavor $f_{A} \ldots f_{2}$ ) in Eq. (15), involved in the hard scattering, and $Z_{S, C D}^{(\mathrm{f})}$ is a matrix of renormalization constants, describing the renormalization of the soft function. 
In the following, we shall adopt the convention of using Roman indexes to refer to the color structures in an arbitrary basis. In jet cross sections near partonic threshold, the hard-scattering function is a product of two purely virtual factors [21]

$$
H_{I L}^{(\mathrm{f})}\left(\frac{M_{J J}}{\mu}, \Delta y, \alpha_{s}\left(\mu^{2}\right)\right)=h_{L}^{(\mathrm{f})^{*}}\left(\frac{M_{J J}}{\mu}, \Delta y, \alpha_{s}\left(\mu^{2}\right)\right) h_{I}^{(\mathrm{f})}\left(\frac{M_{J J}}{\mu}, \Delta y, \alpha_{s}\left(\mu^{2}\right)\right),
$$

one from the overall amplitude and one from the complex conjugate. This simplification [4] is due to the fact that near threshold there is insufficient phase space for the emission of hard partons, aside from those observed in the hard scattering (and hence summarized in the jet functions $J^{(f)}$ in Eq. (29)) .

The $h_{I}$ in Eq. (31) are the coefficients of color tensors in the expansion of the hard scattering amplitude $h_{d_{A} \ldots d_{2}}^{\mathrm{f})}$ treated as a matrix in color space,

$$
h_{d_{A} \ldots d_{2}}^{(\mathrm{f})}\left(\frac{M_{J J}}{\mu}, \Delta y, \alpha_{s}\left(\mu^{2}\right)\right)=\sum_{K} h_{K}^{(\mathrm{f})}\left(\frac{M_{J J}}{\mu}, \Delta y, \alpha_{s}\left(\mu^{2}\right)\right)\left(c_{K}^{(\mathrm{f})}\right)_{d_{A} \ldots d_{2}},
$$

with the $c_{K}^{(\mathrm{f})}$ the same color tensors introduced to define the soft functions, through Eqs. (13), (22) and (28). Here we may point out a strong similarity to the effective theory formalism, in which the $c_{I}$ 's play the role of operators in a "low-energy" theory, and the $h_{I}$ 's are coefficient functions that summarize the high-energy components that have been "integrated out".

From Eq. (30), the soft function $S_{L I}^{(\mathrm{f})}$ satisfies the renormalization group equation [7, 17]

$$
\left(\mu \frac{\partial}{\partial \mu}+\beta(g) \frac{\partial}{\partial g}\right) S_{L I}^{(\mathrm{f})}=-\left(\Gamma_{S}^{(\mathrm{f}) \dagger}\right)_{L B} S_{B I}^{(\mathrm{f})}-S_{L A}^{(\mathrm{f})}\left(\Gamma_{S}^{(\mathrm{f})}\right)_{A I},
$$

where we encounter the same soft anomalous dimension matrix, $\Gamma_{S}^{(\mathrm{f})}$, as in Eq. (16), which is computed directly from the UV divergences of the soft function. We will calculate this matrix for the basic partonic processes $\mathrm{f}$ in a minimal subtraction renormalization scheme, taking $\epsilon=\epsilon_{U V}=4-D$, with $D$ the number of space-time dimensions. The one-loop anomalous dimensions,

$$
\Gamma_{S}^{(\mathrm{f})}(g)=-\frac{g}{2} \frac{\partial}{\partial g} \operatorname{Res}_{\epsilon \rightarrow 0} Z_{S}^{(\mathrm{f})}(g, \epsilon),
$$

are obtained from the residues of the UV poles contained in the matrices of renormalization constants of the soft function.

The moment dependence of the product of the hard and soft functions may now be determined directly from Eq. (33) [21],

$$
\begin{aligned}
\operatorname{Tr}\left\{H ^ { ( \mathrm { f } ) } \left(\frac{M_{J J}}{\mu}, \Delta y\right.\right. & \left.\left., \alpha_{s}\left(\mu^{2}\right)\right) \tilde{S}^{(\mathrm{f})}\left(\frac{M_{J J}}{N \mu}, \Delta y, \alpha_{s}\left(\mu^{2}\right)\right)\right\} \\
= & \operatorname{Tr}\left\{H^{(\mathrm{f})}\left(\frac{M_{J J}}{\mu}, \Delta y, \alpha_{s}\left(\mu^{2}\right)\right)\right. \\
& \times \bar{P} \exp \left[\int_{\mu}^{M_{J J} / N} \frac{d \mu^{\prime}}{\mu^{\prime}} \Gamma_{S}^{(\mathrm{f}) \dagger}\left(\alpha_{s}\left(\mu^{\prime 2}\right)\right)\right]
\end{aligned}
$$




$$
\begin{aligned}
& \times \tilde{S}^{(\mathrm{f})}\left(1, \Delta y, \alpha_{s}\left(M_{J J}^{2} / N^{2}\right)\right) \\
& \left.\times P \exp \left[\int_{\mu}^{M_{J J} / N} \frac{d \mu^{\prime}}{\mu^{\prime}} \Gamma_{S}^{(\mathrm{f})}\left(\alpha_{s}\left(\mu^{\prime 2}\right)\right)\right]\right\},
\end{aligned}
$$

where the symbols $P$ and $\bar{P}$ refer to path-ordering in the same and in the opposite sense as the integration variable $\mu^{\prime} . P$ orders $\Gamma_{S}^{(\mathrm{f})}\left(\alpha_{s}\left(\mu^{2}\right)\right)$ to the far right and $\Gamma_{S}^{(\mathrm{f})}\left(\alpha_{s}\left(M_{J J}^{2} / N^{2}\right)\right)$ to the far left of the products. In Eq. (35) the trace is taken in the basis of color structures, as

$$
\operatorname{Tr}\left\{H^{(\mathrm{f})} \tilde{S}^{(\mathrm{f})}\right\} \equiv H_{I L}^{(\mathrm{f})} \tilde{S}_{L I}^{(\mathrm{f})}=h_{L}^{(\mathrm{f})^{*}} \tilde{S}_{L I}^{(\mathrm{f})} h_{I}^{(\mathrm{f})} .
$$

At lowest order, $\tilde{S}_{L I}^{(\mathrm{f})}$ is just the square of eikonal vertices, with elements given by color traces of $c_{I}$ and $c_{L}^{*}$.

\subsection{Diagonalization of the Color Basis}

In lowest (linear) order in $\alpha_{s}$ for the anomalous dimension matrices, the ordered exponentials of Eq. (35) may be reduced to sums of exponentials, by changing the color basis to one in which $\Gamma_{S}^{(f)}$ is diagonal. We proceed as follows.

First, we treat the original basis of color structures as a set of kets, $\left\{\left|c^{(\mathrm{f})}{ }_{I}\right\rangle\right\}$. Second, we compute the anomalous dimension matrix $\Gamma_{S}^{(\mathrm{f})}$ at one loop in this basis and we then diagonalize it, finding its eigenvalues and eigenvectors. Let the diagonal basis of the eigenvectors be denoted as the set $\left\{\left|e^{(f)}{ }_{\kappa}\right\rangle\right\}$, where from now on we shall use Greek indices for vectors and matrices expressed in this basis. The matrix defined as

$$
\left(R^{(\mathrm{f})}\right)_{K \beta}^{-1} \equiv\left\langle c_{K}^{(\mathrm{f})} \mid e_{\beta}^{(\mathrm{f})}\right\rangle \equiv\left(e^{(\mathrm{f})}{ }_{\beta}\right)_{K}
$$

diagonalizes the anomalous dimension matrix according to the equation

$$
\left[R^{(\mathrm{f})} \Gamma_{S}^{(\mathrm{f})}\left(R^{(\mathrm{f})}\right)^{-1}\right]_{\kappa \beta}=\lambda_{\kappa}^{(\mathrm{f})} \delta_{\kappa \beta}
$$

Third, we reexpress the hard amplitudes in the eigenvector basis. In the previous notation,

$$
\begin{aligned}
\left(h_{\gamma}^{(\mathrm{f})}\right)^{*} & =\left(h_{K}^{(\mathrm{f})}\right)^{*}\left(R^{(\mathrm{f})}\right)_{K \gamma}^{\dagger} \\
h_{\beta}^{(\mathrm{f})} & =\left(R^{(\mathrm{f})}\right)_{\beta L} h_{L}^{(\mathrm{f})} .
\end{aligned}
$$

We reexpress Eq. (36) in the new basis as

$$
\operatorname{Tr}\left\{H^{(\mathrm{f})} \tilde{S}^{(\mathrm{f})}\right\}=\left(h_{\gamma}^{(\mathrm{f})}\right)^{*} \tilde{S}_{\gamma \beta}^{(\mathrm{f})} h_{\beta}^{(\mathrm{f})}=H_{\beta \gamma}^{(\mathrm{f})} \tilde{S}_{\gamma \beta}^{(\mathrm{f})},
$$


where we define

$$
\tilde{S}_{\gamma \beta}^{(\mathrm{f})} \equiv\left[\left(R_{\gamma M}^{(\mathrm{f})}\right)^{-1}\right]^{\dagger} \tilde{S}_{M N}^{(\mathrm{f})}\left(R^{(\mathrm{f})}\right)_{N \beta}^{-1} .
$$

In the diagonal basis of the eigenvectors, Eq. (35) then becomes

$$
\begin{aligned}
\operatorname{Tr}\left\{H ^ { ( \mathrm { f } ) } \left(\frac{M_{J J}}{\mu}\right.\right. & \left.\left., \Delta y, \alpha_{s}\left(\mu^{2}\right)\right) \tilde{S}^{(\mathrm{f})}\left(\frac{M_{J J}}{N \mu}, \Delta y, \alpha_{s}\left(\mu^{2}\right)\right)\right\} \\
= & H_{\beta \gamma}^{(\mathrm{f})}\left(\frac{M_{J J}}{\mu}, \Delta y, \alpha_{s}\left(\mu^{2}\right)\right) \tilde{S}_{\gamma \beta}^{(\mathrm{f})}\left(1, \Delta y, \alpha_{s}\left(M_{J J}^{2} / N^{2}\right)\right) \\
& \times \exp \left\{\int_{\mu}^{M_{J J} / N} \frac{d \mu^{\prime}}{\mu^{\prime}}\left[\lambda_{\gamma}^{(\mathrm{f}) *}\left(\alpha_{s}\left(\mu^{\prime 2}\right)\right)+\lambda_{\beta}^{(\mathrm{f})}\left(\alpha_{s}\left(\mu^{\prime 2}\right)\right)\right]\right\},
\end{aligned}
$$

in which the leading $N$-dependence of the product is organized in a sum of exponentials.

In general, the eigenvalues in Eq. (42) depend on the flavors and directions of the colliding partons, both incoming and outgoing. In the following two sections, for the partonic processes $\mathrm{f}$, we will give:

- The basis of color structures $\left\{\left|c^{(\mathrm{f})} I\right\rangle\right\}$. Generally, we will work with an arbitrary number of colors, $N_{c}$.

- In this basis, the anomalous dimension matrix $\Gamma_{S}^{(\mathrm{f})}$.

- The set of eigenvalues, $\left\{\lambda_{\kappa}^{(\mathrm{f})}\right\}$, and the corresponding set of eigenvectors, $\left\{\left|e^{(\mathrm{f})}\right\rangle\right\rangle$, of $\Gamma_{S}^{(\mathrm{f})}$. Each eigenvector, in the notation of Eq. (37), corresponds to a column of $\left(R^{(\mathrm{f})}\right)^{-1}$.

With these quantities, combined with explicit hard-scattering functions, it is possible to write down resummed jet cross sections [21].

The anomalous dimension matrices for the processes $q \bar{q} \rightarrow q \bar{q}$ and $q q \rightarrow q q$ have

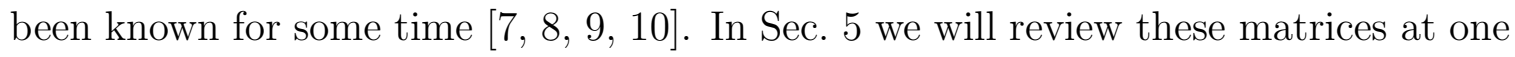
loop, and specify their eigenvectors. There we also give corresponding results for the process $q \bar{q} \rightarrow g g$ (related by time-reversal invariance to $g g \rightarrow q \bar{q}$ as well [17, 18]) and for the process $q g \rightarrow q g$ (related to $\bar{q} g \rightarrow \bar{q} g$ ). The quark-gluon processes involve a $3 \times 3$ matrix, the quark-quark a $2 \times 2$, and all of these results are reasonably straightforward. For gluon-gluon scattering on the other hand, the anomalous dimension matrix is $8 \times 8$ in $S U(3)$, and requires a somewhat more extensive analysis, which we give in Sec. 6.

\section{Anomalous Dimensions for Quark-initiated Scat- tering}




\subsection{Formalism}

Our Wilson lines represent partonic processes, whose momenta and colors are labeled as in Eq. (15), $f_{A}\left(l_{A}, r_{A}\right)+f_{B}\left(l_{B}, r_{B}\right) \rightarrow f_{1}\left(p_{1}, r_{1}\right)+f_{2}\left(p_{2}, r_{2}\right)$. In terms of hadronic momenta, we take $l_{i}=x_{i} p_{i}, i=A, B$, with $x_{i}$ the partonic momentum fraction. Referring to the dijet process Eq. (18) at partonic threshold, the dimensionless, lightlike velocity vectors $v_{i}^{\mu}, v_{i}^{2}=0$, which define the directions of Wilson lines at the vertices $W^{(\mathrm{f})}$, are given by

$$
\begin{aligned}
l_{i}^{\mu} & =M_{J J} v_{i}^{\mu} & & i=A, B \\
p_{i}^{\mu} & =M_{J J} v_{i}^{\mu} & & i=1,2 .
\end{aligned}
$$

We also recall the definitions of partonic Mandelstam invariants,

$$
\begin{aligned}
\hat{s} & =\left(l_{A}+l_{B}\right)^{2} \\
\hat{t} & =\left(l_{A}-p_{1}\right)^{2} \\
\hat{u} & =\left(l_{A}-p_{2}\right)^{2} .
\end{aligned}
$$

The anomalous dimension matrices for all the processes below depend on logarithms of ratios of these invariants, and we introduce for them the following notation:

$$
\begin{aligned}
T & \equiv \ln \left(\frac{-\hat{t}}{\hat{s}}\right)+i \pi \\
U & \equiv \ln \left(\frac{-\hat{u}}{\hat{s}}\right)+i \pi
\end{aligned}
$$

As mentioned above, although the full cross section is gauge independent, the functions into which it is factorized depend, in general, on the choice of $n^{\mu}$, the axial gauge-fixing vector. From the factorized cross section, Eq. (29), it is clear that gauge dependence in the product of $H^{(\mathrm{f})}$ and $S^{(\mathrm{f})}$ must cancel the gauge dependence of the incoming and outgoing jets, $\psi$ and $J^{\left(f_{i}\right)}$. Because the jets are incoherent relative to the hard and soft functions, the gauge dependence of the anomalous dimension matrices $\Gamma_{S}^{(\mathrm{f})}$ must be proportional to the identity matrix. To summarize this gauge dependence we introduce, for each parton in process $\mathrm{f}$, the function

$$
\mathcal{G}_{\left(f_{i}\right)}\left(\nu_{i}\right)=C_{f_{i}} \frac{\alpha_{s}}{\pi}\left[-\frac{1}{2} \ln \left(\nu_{i}\right)-\frac{1}{2} \ln 2+\frac{1}{2}-\frac{1}{2} i \pi\right]
$$

with $C_{f_{i}}=C_{F}\left(C_{A}\right)$ for a quark (gluon), and with (see also Appendix B)

$$
\nu_{i} \equiv \frac{\left(v_{i} \cdot n\right)^{2}}{|n|^{2}} .
$$

In these terms, we will write our anomalous dimension matrix as

$$
\left(\Gamma_{S}^{(\mathrm{f})}\right)_{K L}=\left(\Gamma_{S^{\prime}}^{(\mathrm{f})}\right)_{K L}+\left(\sum_{i=A, B, 1,2} \mathcal{G}_{\left(f_{i}\right)}\left(\nu_{i}\right)\right) \delta_{K L}
$$


and in the following, for specific processes, we will report only $\Gamma_{S^{\prime}}^{(\mathrm{f})}$, to which $\Gamma_{S}^{(\mathrm{f})}$ reduces under a proper choice of gauge. Eq. (48) will allow us to recover the full gauge-dependence, whenever necessary. We emphasize here that the dependence of the anomalous dimension matrix elements on the external eikonal four-vectors and on the axial gauge-fixing vector comes entirely from the evaluation of the graphs shown in Fig. 14, no matter which partonic process we consider. The explicit computation of the graphs shown requires the eikonal Feynman rules, given in Appendix A, and a few one-loop integrations, summarized in Appendix B.

One last remark has to be made about the choice of physical channel $s, t$, or $u$, for the definition of the basis of color structures. All choices are allowed, and related to each other by simple crossing transformations. In the following, apart from the processes $q \bar{q} \rightarrow g g$ and $g g \rightarrow q \bar{q}$, which are better described in terms of $s$-channel color structures, we will always use $t$-channel bases, which seems to be the natural choice when analyzing forward scattering [8]. In the following four subsections we

present the results for the anomalous dimension matrices $\Gamma_{S^{\prime}}^{(\mathrm{f})}$ for partonic processes involving quarks.

\subsection{Soft anomalous dimension for $q \bar{q} \rightarrow q \bar{q}$}

We treat the process

$$
q\left(l_{A}, r_{A}\right)+\bar{q}\left(l_{B}, r_{B}\right) \longrightarrow q\left(p_{1}, r_{1}\right)+\bar{q}\left(p_{2}, r_{2}\right)
$$

in the $t$-channel singlet-octet color basis

$$
\begin{aligned}
c_{1} & =\delta_{r_{A}, r_{1}} \delta_{r_{B}, r_{2}} \\
c_{2} & =-\frac{1}{2 N_{c}} \delta_{r_{A}, r_{1}} \delta_{r_{B}, r_{2}}+\frac{1}{2} \delta_{r_{A}, r_{B}} \delta_{r_{1}, r_{2}} .
\end{aligned}
$$

The procedure we follow is the same as the one described in Refs. [7] and [17, 18]. We evaluate one-loop corrections for each color vertex, as shown in Fig. \&, determining in every case the color decomposition of its ultraviolet divergences. The relevant scalar integrals are reviewed in Appendix B below. The UV divergences found with the color vertex $c_{I}^{(\mathrm{f})}$ in Fig. 4 in general generate counterterms for all the vertices $c_{J}^{(\mathrm{f})}$ in the same set, where $J$ may or may not equal $I$. This is standard operator mixing under renormalization. The elements of the anomalous dimension matrix are then found from Eq. (34).

In this manner, we find the matrix

$$
\Gamma_{S^{\prime}}=\frac{\alpha_{s}}{\pi}\left(\begin{array}{cc}
2 C_{F} T & -\frac{C_{F}}{N_{c}} U \\
-2 U & -\frac{1}{N_{c}}(T-2 U)
\end{array}\right) .
$$

The dependence on the logarithmic ratio $T$ is diagonal in a $t$-channel color basis. Referring to the resummed $N$-dependence in Eq. (42), we see that in the forward region of the partonic scattering $(T \rightarrow-\infty)$ color singlet exchange is exponentially 
enhanced relative to color octet [8]. The eigenvalues of this anomalous dimension matrix are

$$
\begin{aligned}
& \lambda_{1}=\frac{\alpha_{s}}{\pi} \frac{1}{2 N_{c}}\left[\left(N_{c}{ }^{2}-2\right) T+2 U-N_{c} \sqrt{\Delta}\right] \\
& \lambda_{2}=\frac{\alpha_{s}}{\pi} \frac{1}{2 N_{c}}\left[\left(N_{c}{ }^{2}-2\right) T+2 U+N_{c} \sqrt{\Delta}\right],
\end{aligned}
$$

where $\Delta$ is defined as

$$
\Delta=N_{c}^{2} T^{2}-4 T U+4 U^{2}
$$

The corresponding (arbitrarily normalized) eigenvectors are

$$
\begin{aligned}
& e_{1}=\left(\begin{array}{c}
\frac{-N_{c}^{2} T+2 U+N_{c} \sqrt{\Delta}}{4 N_{c} U} \\
1
\end{array}\right) . \\
& e_{2}=\left(\begin{array}{c}
\frac{-N_{c}^{2} T+2 U-N_{c} \sqrt{\Delta}}{4 N_{c} U} \\
1
\end{array}\right) .
\end{aligned}
$$

From these eigenvectors we may reconstruct the matrix $R^{-1}$ of Eq. (37), which gives the transformation from the singlet-octet to the diagonal basis, for any scattering angle. The remaining anomalous dimensions are found in much the same fashion.

\subsection{Soft anomalous dimension for $q q \rightarrow q q$}

In this subsection we analyze the process

$$
q\left(l_{A}, r_{A}\right)+q\left(l_{B}, r_{B}\right) \longrightarrow q\left(p_{1}, r_{1}\right)+q\left(p_{2}, r_{2}\right),
$$

again in the $t$-channel singlet-octet color basis

$$
\begin{aligned}
c_{1} & =-\frac{1}{2 N_{c}} \delta_{r_{A}, r_{1}} \delta_{r_{B}, r_{2}}+\frac{1}{2} \delta_{r_{A}, r_{2}} \delta_{r_{B}, r_{1}} \\
c_{2} & =\delta_{r_{A}, r_{1}} \delta_{r_{B}, r_{2}} .
\end{aligned}
$$

We find the anomalous dimension matrix [8]

$$
\Gamma_{S^{\prime}}=\frac{\alpha_{s}}{\pi}\left(\begin{array}{cc}
-\frac{1}{N_{c}}(T+U)+2 C_{F} U & 2 U \\
\frac{C_{F}}{N_{c}} U & 2 C_{F} T
\end{array}\right) .
$$

Once more, the dependence on the logarithmic ratio $T$ is diagonal in the $t$-channel color basis, and again the color singlet dominates the octet in the forward region. The eigenvalues of this anomalous dimension matrix are

$$
\begin{aligned}
& \lambda_{1}=\frac{\alpha_{s}}{\pi} \frac{1}{2 N_{c}}\left[\left(N_{c}^{2}-2\right)(T+U)-N_{c} \sqrt{\Delta^{\prime}}\right] \\
& \lambda_{2}=\frac{\alpha_{s}}{\pi} \frac{1}{2 N_{c}}\left[\left(N_{c}^{2}-2\right)(T+U)+N_{c} \sqrt{\Delta^{\prime}}\right],
\end{aligned}
$$


where $\Delta^{\prime}$ is defined as

$$
\Delta^{\prime}=N_{c}^{2}(T-U)^{2}+4 T U,
$$

and the corresponding eigenvectors are

$$
\begin{aligned}
& e_{1}=\left(\begin{array}{c}
\frac{-N_{c}{ }^{3}(T-U)-2 N_{c} U-N_{c}{ }^{2} \sqrt{\Delta^{\prime}}}{\left(N_{c}^{2}-1\right) U} \\
1
\end{array}\right) \\
& e_{2}=\left(\begin{array}{c}
\frac{-N_{c}{ }^{3}(T-U)-2 N_{c} U+N_{c} 2 \sqrt{\Delta^{\prime}}}{\left(N_{c}{ }^{2}-1\right) U} \\
1
\end{array}\right) .
\end{aligned}
$$

\subsection{Soft anomalous dimension for $q \bar{q} \rightarrow g g$ and $g g \rightarrow q \bar{q}$}

Next, consider the process

$$
q\left(l_{A}, r_{A}\right)+\bar{q}\left(l_{B}, r_{B}\right) \longrightarrow g\left(p_{1}, r_{1}\right)+g\left(p_{2}, r_{2}\right),
$$

in the $s$-channel color basis

$$
\begin{aligned}
& c_{1}=\delta_{r_{A}, r_{B}} \delta_{r_{1}, r_{2}} \\
& c_{2}=d^{r_{1} r_{2} c}\left(T_{F}^{c}\right)_{r_{B} r_{A}} \\
& c_{3}=i f^{r_{1} r_{2} c}\left(T_{F}^{c}\right)_{r_{B} r_{A}},
\end{aligned}
$$

where the $T_{F}^{c}$ 's are the generators of $S U\left(N_{c}\right)$ in the fundamental representation, while $f^{a b c}$ and $d^{a b c}$ are the totally antisymmetric and symmetric $S U\left(N_{c}\right)$ invariant tensors respectively. In this basis, we find the anomalous dimension matrix

$$
\Gamma_{S^{\prime}}=\frac{\alpha_{s}}{\pi}\left(\begin{array}{ccc}
0 & 0 & U-T \\
0 & \frac{C_{A}}{2}(T+U) & \frac{C_{A}}{2}(U-T) \\
2(U-T) & \frac{N_{c}^{2}-4}{2 N_{c}}(U-T) & \frac{C_{A}}{2}(T+U)
\end{array}\right) .
$$

The same anomalous dimension describes also the time-reversed process [17, 18]

$$
g\left(p_{1}, r_{1}\right)+g\left(p_{2}, r_{2}\right) \longrightarrow \bar{q}\left(l_{A}, r_{A}\right)+q\left(l_{B}, r_{B}\right) .
$$

The eigenvalues of $\Gamma_{S^{\prime}}$ are the solutions of the cubic equation

$$
\begin{array}{r}
\lambda^{3}-\frac{\alpha_{s}}{\pi} C_{A}(T+U) \lambda^{2}+\left(\frac{\alpha_{s}}{\pi}\right)^{2}\left[\left(\frac{C_{A}}{2}(T+U)\right)^{2}-\frac{N_{c}^{2}+4}{4}(U-T)^{2}\right] \lambda \\
+\left(\frac{\alpha_{s}}{\pi}\right)^{3} C_{A}(T+U)(U-T)^{2}=0,
\end{array}
$$

given by

$$
\begin{aligned}
\lambda_{1} & =\frac{\alpha_{s}}{\pi} \frac{1}{3}\left(X^{1 / 3}-Y+C_{A}(T+U)\right) \\
\lambda_{2,3} & =\frac{\alpha_{s}}{\pi} \frac{1}{3}\left[-\frac{1}{2}\left(X^{1 / 3}-Y\right)+C_{A}(T+U) \pm \frac{1}{2} i \sqrt{3}\left(X^{1 / 3}+Y\right)\right],
\end{aligned}
$$


where

$$
\begin{gathered}
X=-\left(\frac{C_{A}}{2}(T+U)\right)^{3}+\frac{9}{8} C_{A}(T+U)(U-T)^{2}\left(N_{c}^{2}-8\right) \\
+\frac{3 \sqrt{3}}{2}\left[-(U-T)^{6} \frac{\left(N_{c}^{2}+4\right)^{3}}{16}-(U-T)^{2}\left(\frac{C_{A}}{2}(T+U)\right)^{4}\left(N_{c}^{2}-4\right)\right. \\
\left.+\frac{1}{2}(U-T)^{4}\left(\frac{C_{A}}{2}(T+U)\right)^{2}\left(\left(N_{c}^{2}-8\right)^{2}-12\left(N_{c}^{2}-2\right)\right)\right]^{1 / 2}
\end{gathered}
$$

and

$$
Y=-\left[\left(\frac{C_{A}}{2}(T+U)\right)^{2}+\frac{3}{4}(U-T)^{2}\left(N_{c}^{2}+4\right)\right] X^{-1 / 3} .
$$

For each eigenvalue $\lambda_{i}$ the corresponding eigenvector is given as

$$
e_{i}=\left(\begin{array}{c}
\frac{U-T}{\lambda_{i}} \\
\frac{N_{c}(U-T)}{2 \lambda_{i}-C_{A}(T+U)} \\
1
\end{array}\right), i=1,2,3
$$

\subsection{Soft anomalous dimension for $q g \rightarrow q g$ and $\bar{q} g \rightarrow \bar{q} g$}

We now turn to the "Compton" process

$$
q\left(l_{A}, r_{A}\right)+g\left(l_{B}, r_{B}\right) \longrightarrow q\left(p_{1}, r_{1}\right)+g\left(p_{2}, r_{2}\right),
$$

in the $t$-channel color basis

$$
\begin{aligned}
c_{1} & =\delta_{r_{A}, r_{1}} \delta_{r_{B}, r_{2}} \\
c_{2} & =d^{r_{B} r_{2} c}\left(T_{F}^{c}\right)_{r_{1} r_{A}} \\
c_{3} & =i f^{r_{B} r_{2} c}\left(T_{F}^{c}\right)_{r_{1} r_{A}}
\end{aligned}
$$

The result of our calculation is

$$
\Gamma_{S^{\prime}}=\frac{\alpha_{s}}{\pi}\left(\begin{array}{ccc}
\left(C_{F}+C_{A}\right) T & 0 & U \\
0 & C_{F} T+\frac{C_{A}}{2} U & \frac{C_{A}}{2} U \\
2 U & \frac{N_{c}^{2}-4}{2 N_{c}} U & C_{F} T+\frac{C_{A}}{2} U
\end{array}\right)
$$

which applies to the process

$$
\bar{q}\left(p_{1}, r_{1}\right)+g\left(p_{2}, r_{2}\right) \longrightarrow \bar{q}\left(l_{A}, r_{A}\right)+g\left(l_{B}, r_{B}\right)
$$

as well. As in Eq. (51), the dependence on the logarithmic ratio $T$ is diagonal in the $t$-channel color basis, and the $t$-channel color singlet dominates in the forward region $(T \rightarrow-\infty)$. 
The eigenvalues have the same structure as in Eq. (66),

$$
\begin{aligned}
\lambda_{1} & =\frac{\alpha_{s}}{\pi} \frac{1}{3}\left(X^{\prime 1 / 3}-Y^{\prime}+\left(3 C_{F}+C_{A}\right) T+C_{A} U\right), \\
\lambda_{2,3} & =\frac{\alpha_{s}}{\pi} \frac{1}{3}\left[-\frac{1}{2}\left(X^{\prime 1 / 3}-Y^{\prime}\right)+\left(3 C_{F}+C_{A}\right) T+C_{A} U \pm \frac{1}{2} i \sqrt{3}\left(X^{\prime 1 / 3}+Y^{\prime}\right)\right],
\end{aligned}
$$

with $X^{\prime}$ and $Y^{\prime}$ given by

$$
\begin{aligned}
X^{\prime}= & \left(C_{A}\left(T-\frac{U}{2}\right)\right)^{3}-\frac{9}{4} C_{A}\left(T-\frac{U}{2}\right) U^{2}\left(N_{c}^{2}-8\right) \\
& +\frac{3 \sqrt{3}}{2}\left[-U^{6} \frac{\left(N_{c}^{2}+4\right)^{3}}{16}-U^{2}\left(C_{A}\left(T-\frac{U}{2}\right)\right)^{4}\left(N_{c}^{2}-4\right)\right. \\
& \left.+\frac{1}{2} U^{4}\left(C_{A}\left(T-\frac{U}{2}\right)\right)^{2}\left(\left(N_{c}^{2}-8\right)^{2}-12\left(N_{c}^{2}-2\right)\right)\right]^{1 / 2}
\end{aligned}
$$

and

$$
Y^{\prime}=-\left[\left(C_{A}\left(T-\frac{U}{2}\right)\right)^{2}+\frac{3}{4} U^{2}\left(N_{c}^{2}+4\right)\right] X^{\prime-1 / 3} .
$$

The eigenvectors have a form similar to Eq. (69),

$$
e_{i}=\left(\begin{array}{c}
\frac{U}{\lambda_{i}-\left(C_{F}+C_{A}\right) T} \\
\frac{N_{c} U}{2 \lambda_{i}-2 C_{F} T-C_{A} U} \\
1
\end{array}\right), i=1,2,3 .
$$

\section{Soft anomalous dimensions for $g g \rightarrow g g$}

In this section, we present the anomalous dimension matrix for the eikonal version of gluon-gluon scattering. Here the proper choice of a set of color vertices is somewhat less obvious. We would like to compute, as easily as possible, the color structure of one-loop corrections. This was not a problem for $2 \rightarrow 2$ partonic processes involving quarks, because the couplings of the fundamental representation are rather simple.

In subsection 6.1 below, we introduce a basis of nine color tensors [28, 29], which, although overcomplete, is convenient for computation of the one-loop $\Gamma_{S^{\prime}}$ for the gluon-gluon case. We give a few details of how to color-decompose the one-loop graphs into this set, and present a first, "raw" version of the anomalous dimension matrix $\Gamma_{S^{\prime}}$. In subsection 6.2, we modify the original basis, to one in which $\Gamma_{S^{\prime}}$ is in a block-diagonal form in which one block is diagonalized. We also present eigenvalues and eigenvectors in this basis. Finally, in subsection 6.3, working with $N_{c}=3$, we recall $t$-channel color projectors describing the decomposition of the product of two color octets into irreducible representations [30]. We use these projectors to reduce our set of color structures to a complete, eight-dimensional basis of color tensors. We exhibit the resulting one-loop anomalous dimension matrix, along with its eigenvalues and eigenvectors. As in all cases above, logarithms of $t / s$ appear only in the diagonal of $\Gamma_{S^{\prime}}$ in this basis. 


\subsection{Initial basis and $\Gamma_{S^{\prime}}$ at one loop}

To begin with, we consider the set of color tensors, in terms of which an arbitrary four-gluon diagram, with external color indices $r_{A}, r_{B}, r_{1}$ and $r_{2}$ can be expanded 28, 29

$$
\begin{aligned}
& c_{1}=\operatorname{Tr}\left(T_{F}^{r_{A}} T_{F}^{r_{B}} T_{F}^{r_{2}} T_{F}^{r_{1}}\right), \\
& c_{2}=\operatorname{Tr}\left(T_{F}^{r_{A}} T_{F}^{r_{B}} T_{F}^{r_{1}} T_{F}^{r_{2}}\right), \\
& c_{3}=\operatorname{Tr}\left(T_{F}^{r_{A}} T_{F}^{r_{1}} T_{F}^{r_{2}} T_{F}^{r_{B}}\right), \\
& c_{4}=\operatorname{Tr}\left(T_{F}^{r_{A}} T_{F}^{r_{1}} T_{F}^{r_{B}} T_{F}^{r_{2}}\right), \\
& c_{5}=\operatorname{Tr}\left(T_{F}^{r_{A}} T_{F}^{r_{2}} T_{F}^{r_{1}} T_{F}^{r_{B}}\right), \\
& c_{6}=\operatorname{Tr}\left(T_{F}^{r_{A}} T_{F}^{r_{2}} T_{F}^{r_{B}} T_{F}^{r_{1}}\right), \\
& c_{7}=\frac{1}{4} \delta_{r_{A} r_{1}} \delta_{r_{B} r_{2}}, \\
& c_{8}=\frac{1}{4} \delta_{r_{A} r_{B}} \delta_{r_{1} r_{2}}, \\
& c_{9}=\frac{1}{4} \delta_{r_{A} r_{2}} \delta_{r_{B} r_{1},},
\end{aligned}
$$

where the $T_{F}^{r_{i}}$ s s are the generators of $S U\left(N_{c}\right)$ in the fundamental representation. This set is illustrated graphically in Fig. 5, where all the lines and vertices have only color content. The correspondence between the picture and Eq. (78) is straightforward, in terms of the color dependence of the quark-gluon vertex in the QCD Lagrangian.

As in the case of scattering represented by Wilson lines in the fundamental representation, there is no mixing of operators with different numbers of Wilson lines. Therefore we only need to consider color tensors with four indices in the adjoint representation. Eq. (78) clearly includes the maximum number of inequivalent traces we can build out of four generators $T_{F}^{r_{i}}$, in addition to the three possible singlet combinations. The set (78) mixes only with itself under renormalization. To see this, consider one-loop corrections to the vertices of Eq. (78). The color decomposition of these one-loop corrections is obtained by the graphical identities shown in Fig. 6, whose analytic form is

$$
\begin{aligned}
& f^{r_{1} r_{2} r_{3}}=-2 i\left[\operatorname{Tr}\left(T_{F}^{r_{1}} T_{F}^{r_{2}} T_{F}^{r_{3}}\right)-\operatorname{Tr}\left(T_{F}^{r_{1}} T_{F}^{r_{3}} T_{F}^{r_{2}}\right)\right] \\
& \sum_{k}\left[\left(T_{F}^{k}\right)_{r_{3} r_{1}}\left(T_{F}^{k}\right)_{r_{4} r_{2}}\right]=\frac{1}{2} \delta_{r_{4} r_{1}} \delta_{r_{3} r_{2}}-\frac{1}{2 N_{c}} \delta_{r_{3} r_{1}} \delta_{r_{4} r_{2}},
\end{aligned}
$$

where $f^{r_{1} r_{2} r_{3}}$ are the structure constants of the $S U\left(N_{c}\right)$ algebra. The first identity allows us to replace three-gluon couplings by color traces in the fundamental representation, and the second to "expand" the color content of internal gluons and Wilson lines into pairs of color lines in the fundamental representation. External gluon lines may be treated in the same way, by use of the identity $\delta_{a b}=2 \operatorname{Tr}\left[T_{F}^{a} T_{F}^{b}\right]$. It is straightforward to extend this procedure iteratively to an arbitrary order, using the corresponding identities for the four-point function. Essentially the same color decomposition can be used to show that the basis (78) is complete in the expansion of the hard-scattering function for gluon-gluon scattering, as in Eq. (32) above. 
As in Sec. 5, we follow the procedure of Refs. [7] and [17, 18], and compute the anomalous dimensions from the color decomposition into the set (78) of the one-loop ultraviolet divergences of Fig. 14, using Eq. (34). The choice of basis in Eq. (78) is particularly well-adapted to this procedure, when we employ the identities of Eq. (79). After straightforward, although rather tedious, calculations, we find the $9 \times 9$ anomalous dimension matrix,

$$
\Gamma_{S^{\prime}}=\frac{\alpha_{s} C_{A}}{\pi}\left(\begin{array}{ccccccccc}
T & 0 & 0 & 0 & 0 & 0 & -\frac{U}{N_{c}} & \frac{T-U}{N_{c}} & 0 \\
0 & U & 0 & 0 & 0 & 0 & 0 & \frac{U-T}{N_{c}} & -\frac{T}{N_{c}} \\
0 & 0 & T & 0 & 0 & 0 & -\frac{U}{N_{c}} & \frac{T-U}{N_{c}} & 0 \\
0 & 0 & 0 & (T+U) & 0 & 0 & \frac{U}{N_{c}} & 0 & \frac{T}{N_{c}} \\
0 & 0 & 0 & 0 & U & 0 & 0 & \frac{U-T}{N_{c}} & -\frac{T}{N_{c}} \\
0 & 0 & 0 & 0 & 0 & (T+U) & \frac{U}{N_{c}} & 0 & \frac{T}{N_{c}} \\
\frac{T-U}{N_{c}} & 0 & \frac{T-U}{N_{c}} & \frac{T}{N_{c}} & 0 & \frac{T}{N_{c}} & 2 T & 0 & 0 \\
-\frac{U}{N_{c}} & -\frac{T}{N_{c}} & -\frac{U}{N_{c}} & 0 & -\frac{T}{N_{c}} & 0 & 0 & 0 & 0 \\
0 & \frac{U-T}{N_{c}} & 0 & \frac{U}{N_{c}} & \frac{U-T}{N_{c}} & \frac{U}{N_{c}} & 0 & 0 & 2 U
\end{array}\right) .
$$

Interestingly, this anomalous dimension matrix is diagonal in the large $N_{c}$ limit.

\subsection{Block-diagonal form of the anomalous dimension matrix: eigenvalues and eigenvectors}

Although it has many zeros, the anomalous dimension matrix of Eq. (80) is not transparently easy to diagonalize for arbitrary $N_{c}$. As a first step in its simplification, we will make a change of color basis that transforms (80) into a block-diagonal form. In particular, looking at the first three rows of the matrix, we notice that, if we are able to eliminate the non-zero off-diagonal entries, we immediately determine three of the eigenvalues.

The way to accomplish our purpose is to employ symmetric and antisymmetric linear transformations from the set (78) to a new set $\left\{c_{I}^{\prime}\right\}$,

$$
\begin{aligned}
& c_{1}^{\prime}=\operatorname{Tr}\left(T_{F}^{r_{A}} T_{F}^{r_{B}} T_{F}^{r_{2}} T_{F}^{r_{1}}\right)-\operatorname{Tr}\left(T_{F}^{r_{A}} T_{F}^{r_{1}} T_{F}^{r_{2}} T_{F}^{r_{B}}\right)=c_{1}-c_{3}, \\
& c_{2}^{\prime}=\operatorname{Tr}\left(T_{F}^{r_{A}} T_{F}^{r_{B}} T_{F}^{r_{1}} T_{F}^{r_{2}}\right)-\operatorname{Tr}\left(T_{F}^{r_{A}} T_{F}^{r_{2}} T_{F}^{r_{1}} T_{F}^{r_{B}}\right)=c_{2}-c_{5}, \\
& c_{3}^{\prime}=\operatorname{Tr}\left(T_{F}^{r_{A}} T_{F}^{r_{1}} T_{F}^{r_{B}} T_{F}^{r_{2}}\right)-\operatorname{Tr}\left(T_{F}^{r_{A}} T_{F}^{r_{2}} T_{F}^{r_{B}} T_{F}^{r_{1}}\right)=c_{4}-c_{6}, \\
& c_{4}^{\prime}=\operatorname{Tr}\left(T_{F}^{r_{A}} T_{F}^{r_{B}} T_{F}^{r_{2}} T_{F}^{r_{1}}\right)+\operatorname{Tr}\left(T_{F}^{r_{A}} T_{F}^{r_{1}} T_{F}^{r_{2}} T_{F}^{r_{B}}\right)=c_{1}+c_{3}, \\
& c_{5}^{\prime}=\operatorname{Tr}\left(T_{F}^{r_{A}} T_{F}^{r_{B}} T_{F}^{r_{1}} T_{F}^{r_{2}}\right)+\operatorname{Tr}\left(T_{F}^{r_{A}} T_{F}^{r_{2}} T_{F}^{r_{1}} T_{F}^{r_{B}}\right)=c_{2}+c_{5}, \\
& c_{6}^{\prime}=\operatorname{Tr}\left(T_{F}^{r_{A}} T_{F}^{r_{1}} T_{F}^{r_{B}} T_{F}^{r_{2}}\right)+\operatorname{Tr}\left(T_{F}^{r_{A}} T_{F}^{r_{2}} T_{F}^{r_{B}} T_{F}^{r_{1}}\right)=c_{4}+c_{6}, \\
& c_{7}^{\prime}=\frac{1}{4} \delta_{r_{A} r_{1}} \delta_{r_{B} r_{2}}=c_{7}, \\
& c_{8}^{\prime}=\frac{1}{4} \delta_{r_{A} r_{B}} \delta_{r_{1} r_{2}}=c_{8},
\end{aligned}
$$




$$
c_{9}^{\prime}=\frac{1}{4} \delta_{r_{A} r_{2}} \delta_{r_{B} r_{1}}=c_{9}
$$

The expression of $\Gamma_{S^{\prime}}$ in this new set of color structures has the form

$$
\Gamma_{S^{\prime}}=\left(\begin{array}{cc}
\Gamma_{3 \times 3} & 0_{3 \times 6} \\
0_{6 \times 3} & \Gamma_{6 \times 6}
\end{array}\right),
$$

where the block $\Gamma_{3 \times 3}$ is diagonal,

$$
\Gamma_{3 \times 3}=\frac{\alpha_{s}}{\pi}\left(\begin{array}{ccc}
N_{c} T & 0 & 0 \\
0 & N_{c} U & 0 \\
0 & 0 & N_{c}(T+U)
\end{array}\right),
$$

while the block $\Gamma_{6 \times 6}$ is given by

$$
\Gamma_{6 \times 6}=\frac{\alpha_{s}}{\pi}\left(\begin{array}{cccccc}
N_{c} T & 0 & 0 & -U & T-U & 0 \\
0 & N_{c} U & 0 & 0 & U-T & -T \\
0 & 0 & N_{c}(U+T) & U & 0 & T \\
2(T-U) & 0 & 2 T & 2 N_{c} T & 0 & 0 \\
-2 U & -2 T & 0 & 0 & 0 & 0 \\
0 & -2(T-U) & 2 U & 0 & 0 & 2 N_{c} U
\end{array}\right) .
$$

In both the previous matrices we have used explicitly $C_{A}=N_{c}$ (compare with (80)).

The first three eigenvalues, $\lambda_{1}, \lambda_{2}$ and $\lambda_{3}$, can be read directly from Eq. (83). To determine the others, we just need the diagonalization of a $6 \times 6$ matrix. We have computed its eigenvalues and obtained

$$
\begin{aligned}
\lambda_{4} & =\frac{\alpha_{s}}{\pi} N_{c} T=\lambda_{1} \\
\lambda_{5} & =\frac{\alpha_{s}}{\pi} N_{c} U=\lambda_{2}, \\
\lambda_{6} & =\frac{\alpha_{s}}{\pi} N_{c}(T+U)=\lambda_{3}, \\
\lambda_{7} & =\frac{\alpha_{s}}{\pi}\left[X^{1 / 3}-Y+\frac{2}{3} N_{c}(T+U)\right] \\
\lambda_{8,9} & =\frac{\alpha_{s}}{\pi}\left[-\frac{1}{2}\left(X^{1 / 3}-Y\right)+\frac{2}{3} N_{c}(T+U) \pm \frac{1}{2} i \sqrt{3}\left(X^{1 / 3}+Y\right)\right],
\end{aligned}
$$

where, as before, to reduce clutter in the notation, we introduce auxiliary quantities $X$ and $Y$, defined for this particular case as

$$
\begin{aligned}
X= & \frac{4}{27} N_{c}\left(N_{c}^{2}-9\right)\left[2\left(T^{3}+U^{3}\right)-3\left(T^{2} U+T U^{2}\right)\right] \\
& +\frac{4}{9}\left\{-12\left(N_{c}^{2}-1\right)^{2}\left[T^{6}+U^{6}-3\left(T^{5} U+T U^{5}\right)\right]\right. \\
& -3\left(N_{c}^{6}+6 N_{c}^{4}+33 N_{c}^{2}+24\right)\left(T^{4} U^{2}+T^{2} U^{4}\right) \\
& \left.+6 T^{3} U^{3}\left(N_{c}^{6}-4 N_{c}^{4}+53 N_{c}^{2}+14\right)\right\}^{1 / 2}
\end{aligned}
$$


and

$$
Y=-\frac{4}{9}\left(N_{c}^{2}+3\right)\left[T^{2}+U^{2}-T U\right] X^{-1 / 3}
$$

The eigenvectors corresponding to the first three eigenvalues have the form

$$
e_{i}=\left(\begin{array}{c}
e_{i}^{(3)} \\
0^{(6)}
\end{array}\right), i=1,2,3
$$

where the superscripts refer to the dimension. Thus, the $e_{i}^{(3)}$ 's are three-dimensional vectors, defined as

$$
e_{i}^{(3)}=\left(\begin{array}{c}
\delta_{i 1} \\
\delta_{i 2} \\
\delta_{i 3}
\end{array}\right), i=1,2,3,
$$

while $0^{(6)}$ is the six-dimensional null vector.

The eigenvectors corresponding to the other eigenvalues have the general form

$$
e_{i}=\left(\begin{array}{c}
0^{(3)} \\
e_{i}^{(6)}
\end{array}\right), i=4, \ldots, 9
$$

In particular $e_{4}^{(6)}, e_{5}^{(6)}$ and $e_{6}^{(6)}$ are given by

$$
e_{4}^{(6)}=\left(\begin{array}{c}
\frac{\left(N_{c}^{2}-2\right) T}{2 U} \\
1 \\
\frac{T-U}{U} \\
N_{c} \frac{U-T}{U} \\
-N_{c} \\
0
\end{array}\right), \quad e_{5}^{(6)}=\left(\begin{array}{c}
-\frac{1}{N_{c}} \\
-\frac{\left(N_{c}^{2}-2\right) U}{2 N_{c} T} \\
\frac{T-U}{T N_{c}} \\
0 \\
1 \\
\frac{U-T}{T}
\end{array}\right), \quad e_{6}^{(6)}=\left(\begin{array}{c}
-\frac{T}{U} \\
1 \\
\left(N_{c}^{2}-2\right) \frac{U-T}{2 U} \\
N_{c} \frac{T}{U} \\
0 \\
-N_{c}
\end{array}\right)
$$

while $e_{7}^{(6)}, e_{8}^{(6)}$ and $e_{9}^{(6)}$ are given in terms of the coefficients of $\alpha_{s} / \pi$ in the corresponding eigenvalues,

$$
\lambda_{i}^{\prime}=\frac{\pi}{\alpha_{s}} \lambda_{i}
$$

by

$$
e_{i}^{(6)}=\left(\begin{array}{c}
x_{1}\left(\lambda_{i}^{\prime}\right) \\
x_{2}\left(\lambda_{i}^{\prime}\right) \\
x_{3}\left(\lambda_{i}^{\prime}\right) \\
x_{4}\left(\lambda_{i}^{\prime}\right) \\
1 \\
x_{6}\left(\lambda_{i}^{\prime}\right)
\end{array}\right), i=7,8,9
$$

Here, once more to shorten our rather complicated formulas, we have parametrized the eigenvectors in terms of the quantities

$$
x_{1}\left(\lambda_{i}^{\prime}\right)=\frac{1}{2 U}\left\{-\lambda_{i}^{\prime}+T \frac{4 N_{c}\left(T^{2}-U^{2}\right)-2 \lambda_{i}^{\prime}(2 T-U)}{\left[N_{c}(T+U)-\lambda_{i}^{\prime}\right]\left(\lambda_{i}^{\prime}-2 N_{c} U\right)}\right\},
$$




$$
\begin{aligned}
x_{2}\left(\lambda_{i}^{\prime}\right)= & -\frac{1}{2 T}\left[\lambda_{i}^{\prime}+2 U x_{1}\left(\lambda_{i}^{\prime}\right)\right], \\
x_{3}\left(\lambda_{i}^{\prime}\right)= & \frac{1}{4 U T^{2}}\left\{\left[\lambda_{i}^{\prime}+2 U x_{1}\left(\lambda_{i}^{\prime}\right)\right]\left[\left(\lambda_{i}^{\prime}-2 N_{c} U\right)\left(\lambda_{i}^{\prime}-N_{c} U\right)-2 T(T-U)\right]\right. \\
& \left.-2 T(T-U)\left(\lambda_{i}^{\prime}-2 N_{c} U\right)\right\}, \\
x_{4}\left(\lambda_{i}^{\prime}\right)= & \frac{1}{U}\left[\left(N_{c} T-\lambda_{i}^{\prime}\right) x_{1}\left(\lambda_{i}^{\prime}\right)+T-U\right], \\
x_{6}\left(\lambda_{i}^{\prime}\right)= & \frac{1}{T}\left\{\frac{\left(\lambda_{i}^{\prime}-N_{c} U\right)\left[\lambda_{i}^{\prime}+2 U x_{1}\left(\lambda_{i}^{\prime}\right)\right]}{2 T}+U-T\right\} .
\end{aligned}
$$

Working with $N_{c}=3$, the eigenvalues simplify to

$$
\begin{aligned}
\lambda_{1} & =\lambda_{4}=3 \frac{\alpha_{s}}{\pi} T, \\
\lambda_{2} & =\lambda_{5}=3 \frac{\alpha_{s}}{\pi} U, \\
\lambda_{3} & =\lambda_{6}=3 \frac{\alpha_{s}}{\pi}(T+U), \\
\lambda_{7} & =2 \frac{\alpha_{s}}{\pi}(T+U), \\
\lambda_{8,9} & =\frac{\alpha_{s}}{\pi}\left[2 T+2 U \pm 4 \sqrt{T^{2}+U^{2}-T U}\right] .
\end{aligned}
$$

For $N_{c}=3$, the eigenvectors $e_{4}^{(6)}, e_{5}^{(6)}$ and $e_{6}^{(6)}$ become (compare Eq. (91))

$$
e_{4}^{(6)}=\left(\begin{array}{c}
-\frac{7 T}{6 U} \\
-\frac{1}{3} \\
\frac{U-T}{3 U} \\
\frac{T-U}{U} \\
1 \\
0
\end{array}\right), \quad e_{5}^{(6)}=\left(\begin{array}{c}
\frac{1}{3} \frac{T}{T-U} \\
\frac{7 U}{6(T-U)} \\
-\frac{1}{3} \\
0 \\
-\frac{T}{T-U} \\
1
\end{array}\right), \quad e_{6}^{(6)}=\left(\begin{array}{c}
\frac{T}{3 U} \\
-\frac{1}{3} \\
\frac{7(T-U)}{6 U} \\
-\frac{T}{U} \\
0 \\
1
\end{array}\right),
$$

while $e_{7}^{(6)}, e_{8}^{(6)}$ and $e_{9}^{(6)}$ reduce to

$$
e_{7}^{(6)}=\left(\begin{array}{c}
-1 \\
-1 \\
-1 \\
1 \\
1 \\
1
\end{array}\right), \quad e_{i}=\left(\begin{array}{c}
a_{1}\left(\lambda_{i}^{\prime}\right) \\
a_{2}\left(\lambda_{i}^{\prime}\right) \\
a_{3}\left(\lambda_{i}^{\prime}\right) \\
a_{4}\left(\lambda_{i}^{\prime}\right) \\
1 \\
a_{6}\left(\lambda_{i}^{\prime}\right)
\end{array}\right), i=8,9
$$

Here for brevity we have introduced the quantities

$$
\begin{aligned}
& a_{1}\left(\lambda_{i}^{\prime}\right)=\frac{-1}{6 U K}\left[20\left(T^{3}-U^{3}\right)-52 T U(T-U)+\left(10 T^{2}+5 U^{2}-9 T U\right) \lambda_{i}^{\prime}\right], \\
& a_{2}\left(\lambda_{i}^{\prime}\right)=a_{1}\left(\lambda_{i}^{\prime}, T \leftrightarrow U\right),
\end{aligned}
$$




$$
\begin{aligned}
a_{3}\left(\lambda_{i}^{\prime}\right)= & \frac{1}{6 T U K}\left\{-36 T U\left(T^{2}+U^{2}\right)+32 T^{2} U^{2}+20\left(T^{4}+U^{4}\right)\right. \\
& \left.+\left[10\left(T^{3}+U^{3}\right)+5 T U(T+U)\right] \lambda_{i}^{\prime}\right\}, \\
a_{4}\left(\lambda_{i}^{\prime}\right)= & \frac{1}{2 U^{2} K}\left[10 U^{4}+20 T^{4}-54 T^{3} U+58 T^{2} U^{2}-34 T U^{3}\right. \\
& \left.+\left(10 T^{2}+7 U^{2}-7 T U\right) T \lambda_{i}^{\prime}\right], \\
a_{6}\left(\lambda_{i}^{\prime}\right)= & a_{4}\left(\lambda_{i}^{\prime}, T \leftrightarrow U\right),
\end{aligned}
$$

where the $\lambda_{i}^{\prime}$ 's are obtained from the $\lambda_{i}$ 's of (95) according to Eq. (92). The quantity $K$ is defined as

$$
K \equiv 5\left(T^{2}+U^{2}\right)-6 U T \text {. }
$$

Using the formalism of subsection 4.2, we can write the matrix $\left(R^{(\mathrm{f})}\right)^{-1}$, having the eigenvectors of the anomalous dimension matrix in its columns. We have verified that $R^{(\mathrm{f})} \Gamma_{S^{\prime}}\left(R^{(\mathrm{f})}\right)^{-1}$ is a diagonal matrix with the eigenvalues of Eq. (95).

\subsection{Color projections for $g g \rightarrow g g$}

So far we have been working with the sets of color structures in Eqs. (78) and (81). These sets are both overcomplete, since the $c_{I}$ 's are not linearly independent [28], as we will show below. In this section we would like to rewrite the set in Eq. (81) in terms of $S U(3)$ tensors (we keep $N_{c}=3$ ), and to elucidate the group structure of $t$-channel color exchange for the product of Wilson lines that generates the same noncollinear soft radiation as $g g \rightarrow g g$.

To relate the basis of traces to color exchange, we first recall the product formula for generators of $S U\left(N_{c}\right)$ in the fundamental representation,

$$
T_{F}^{i} T_{F}^{j}=\frac{1}{6} \delta_{i j} 1+\frac{1}{2}\left(d_{i j k}+i f_{i j k}\right) T_{F}^{k} .
$$

Eq. (100) enables us to rewrite the set of color structures in Eq. (81) in terms of the tensors $f$ and $d$,

$$
\begin{aligned}
& c^{\prime}{ }_{1}=\operatorname{Tr}\left(T_{F}^{r_{A}} T_{F}^{r_{B}} T_{F}^{r_{2}} T_{F}^{r_{1}}\right)-\operatorname{Tr}\left(T_{F}^{r_{A}} T_{F}^{r_{1}} T_{F}^{r_{2}} T_{F}^{r_{B}}\right)=\frac{i}{4}\left[f_{r_{A} r_{B} l} d_{r_{1} r_{2} l}-d_{r_{A} r_{B} l} f_{r_{1} r_{2} l}\right], \\
& c^{\prime}{ }_{2}=\operatorname{Tr}\left(T_{F}^{r_{A}} T_{F}^{r_{B}} T_{F}^{r_{1}} T_{F}^{r_{2}}\right)-\operatorname{Tr}\left(T_{F}^{r_{A}} T_{F}^{r_{2}} T_{F}^{r_{1}} T_{F}^{r_{B}}\right)=\frac{i}{4}\left[f_{r_{A} r_{B} l} d_{r_{1} r_{2} l}+d_{r_{A} r_{B} l} f_{r_{1} r_{2} l}\right], \\
& c^{\prime}{ }_{3}=\operatorname{Tr}\left(T_{F}^{r_{A}} T_{F}^{r_{1}} T_{F}^{r_{B}} T_{F}^{r_{2}}\right)-\operatorname{Tr}\left(T_{F}^{r_{A}} T_{F}^{r_{2}} T_{F}^{r_{B}} T_{F}^{r_{1}}\right)=\frac{i}{4}\left[f_{r_{A} r_{1} l} d_{r_{B} r_{2} l}+d_{r_{A} r_{1} l} f_{r_{B} r_{2} l}\right], \\
& c^{\prime}{ }_{4}=\operatorname{Tr}\left(T_{F}^{r_{A}} T_{F}^{r_{B}} T_{F}^{r_{2}} T_{F}^{r_{1}}\right)+\operatorname{Tr}\left(T_{F}^{r_{A}} T_{F}^{r_{1}} T_{F}^{r_{2}} T_{F}^{r_{B}}\right)=\frac{1}{6} \delta_{r_{A} r_{1}} \delta_{r_{B} r_{2}}+\frac{1}{4}\left[d_{r_{A} r_{1} l} d_{r_{B} r_{2} l}\right. \\
& \left.+f_{r_{A} r_{1} l} f_{r_{B} r_{2} l}\right] \\
& c^{\prime}{ }_{5}=\operatorname{Tr}\left(T_{F}^{r_{A}} T_{F}^{r_{B}} T_{F}^{r_{1}} T_{F}^{r_{2}}\right)+\operatorname{Tr}\left(T_{F}^{r_{A}} T_{F}^{r_{2}} T_{F}^{r_{1}} T_{F}^{r_{B}}\right)=\frac{1}{6} \delta_{r_{A} r_{B}} \delta_{r_{1} r_{2}}+\frac{1}{4}\left[d_{r_{A} r_{B} l} d_{r_{1} r_{2} l}\right. \\
& \left.-f_{r_{A} r_{B} l} f_{r_{1} r_{2} l}\right] \\
& c^{\prime}{ }_{6}=\operatorname{Tr}\left(T_{F}^{r_{A}} T_{F}^{r_{1}} T_{F}^{r_{B}} T_{F}^{r_{2}}\right)+\operatorname{Tr}\left(T_{F}^{r_{A}} T_{F}^{r_{2}} T_{F}^{r_{B}} T_{F}^{r_{1}}\right)=\frac{1}{6} \delta_{r_{A} r_{1}} \delta_{r_{B} r_{2}}+\frac{1}{4}\left[d_{r_{A} r_{1} l} d_{r_{B} r_{2} l}\right.
\end{aligned}
$$




$$
\begin{aligned}
c_{7}^{\prime} & =\frac{1}{4} \delta_{r_{A} r_{1}} \delta_{r_{B} r_{2}}, \\
c^{\prime} & =\frac{1}{4} \delta_{r_{A} r_{B}} \delta_{r_{1} r_{2}}, \\
c^{\prime} & =\frac{1}{4} \delta_{r_{A} r_{2}} \delta_{r_{B} r_{1}} .
\end{aligned}
$$

We now go on to discuss color in the $t$-channel.

The color content of a set of two gluons in $S U(3)$ is described by the direct product $8 \otimes 8$, which can be decomposed into irreducible representations in the standard way, as

$$
8 \otimes 8=1+8_{S}+8_{A}+10+\overline{10}+27 .
$$

In Ref. [30] we can find the representation in terms of $S U(3)$ tensors of the projectors performing the above decomposition. In our notation, $t$-channel projectors are given by

$$
\begin{aligned}
P_{1}\left(r_{A}, r_{B} ; r_{1}, r_{2}\right)= & \frac{1}{8} \delta_{r_{A} r_{1}} \delta_{r_{B} r_{2}}, \\
P_{8_{S}}\left(r_{A}, r_{B} ; r_{1}, r_{2}\right)= & \frac{3}{5} d_{r_{A} r_{1} c} d_{r_{B} r_{2} c}, \\
P_{8_{A}}\left(r_{A}, r_{B} ; r_{1}, r_{2}\right)= & \frac{1}{3} f_{r_{A} r_{1} c} f_{r_{B} r_{2} c}, \\
P_{10+\overline{10}}\left(r_{A}, r_{B} ; r_{1}, r_{2}\right)= & \frac{1}{2}\left(\delta_{r_{A} r_{B}} \delta_{r_{1} r_{2}}-\delta_{r_{A} r_{2}} \delta_{r_{B} r_{1}}\right)-\frac{1}{3} f_{r_{A} r_{1} c} f_{r_{B} r_{2} c} \\
P_{27}\left(r_{A}, r_{B} ; r_{1}, r_{2}\right)= & \frac{1}{2}\left(\delta_{r_{A} r_{B}} \delta_{r_{1} r_{2}}+\delta_{r_{A} r_{2}} \delta_{r_{B} r_{1}}\right)-\frac{1}{8} \delta_{r_{A} r_{1}} \delta_{r_{B} r_{2}} \\
& -\frac{3}{5} d_{r_{A} r_{1} c} d_{r_{B} r_{2} c} .
\end{aligned}
$$

From a comparison of Eq. (101) and Eq. (103), we see that the first three elements of the basis (101), depending only on the "mixed" combinations $f d, d f$, cannot be expressed in terms of these projectors. On the other hand, it is easy to find relations for the remaining basis tensors,

$$
\begin{aligned}
c^{\prime} & =\frac{4}{3} P_{1}+\frac{5}{12} P_{8_{S}}+\frac{3}{4} P_{8_{A}}, \\
c^{\prime}{ }_{5} & =-\frac{1}{6} P_{1}-\frac{1}{3} P_{8_{S}}+\frac{1}{2} P_{27}, \\
c^{\prime} & =\frac{4}{3} P_{1}+\frac{5}{12} P_{8_{S}}-\frac{3}{4} P_{8_{A}}, \\
c_{7}^{\prime} & =2 P_{1}, \\
c^{\prime} & =\frac{1}{4}\left(P_{1}+P_{8_{A}}+P_{8_{S}}+P_{10 \oplus \overline{10}}+P_{27}\right), \\
c_{9}^{\prime} & =\frac{1}{4}\left(P_{1}-P_{8_{A}}+P_{8_{S}}-P_{10 \oplus \overline{10}}+P_{27}\right) .
\end{aligned}
$$


Evidently, the six tensors $c_{I}^{\prime}, i=4 \ldots 9$ may be replaced by only five $t$-channel projectors. This is because, as pointed out above, the original basis of Eq. (78) is overcomplete, and indeed, the basis elements of Eq. (81) satisfy the relation

$$
c^{\prime}{ }_{4}+c_{5}^{\prime}+c_{6}^{\prime}=c_{7}^{\prime}+c_{8}^{\prime}+c_{9}^{\prime} .
$$

In terms of the invariant tensor $d_{i j k}$, related to the $c_{I}^{\prime}$ in Eq. (101), this is equivalent to the identity [28]

$$
d_{i \ell m} d_{m j k}+d_{j \ell m} d_{i m k}+d_{k \ell m} d_{i j m}=\frac{1}{3}\left(\delta_{i j} \delta_{k \ell}+\delta_{j k} \delta_{i \ell}+\delta_{i k} \delta_{j \ell}\right) .
$$

It may be worth noting that the Jacobi identity, as well as a related identity involving products of $f$ 's and d's in Ref. [28], is simply an expression of the cyclicity of traces in the original basis, Eq. (78), and hence does not result in a further reduction of its dimension.

Our new basis is thus given by the eight color structures

$$
\left\{c_{1}^{\prime}, c_{2}^{\prime}, c_{3}^{\prime}, P_{1}, P_{8_{S}}, P_{8_{A}}, P_{10 \oplus \overline{10}}, P_{27}\right\} .
$$

In this basis, the soft anomalous dimension matrix becomes

$$
\Gamma_{S^{\prime}}=\left(\begin{array}{cc}
\Gamma_{3 \times 3} & 0_{3 \times 5} \\
0_{5 \times 3} & \Gamma_{5 \times 5}
\end{array}\right),
$$

with $\Gamma_{3 \times 3}$ given by Eq. (83) with $N_{c}=3$, and with $\Gamma_{5 \times 5}$ given by

$$
\Gamma_{5 \times 5}=\frac{\alpha_{s}}{\pi}\left(\begin{array}{ccccc}
6 T & 0 & -6 U & 0 & 0 \\
0 & 3 T+\frac{3 U}{2} & -\frac{3 U}{2} & -3 U & 0 \\
-\frac{3 U}{4} & -\frac{3 U}{2} & 3 T+\frac{3 U}{2} & 0 & -\frac{9 U}{4} \\
0 & -\frac{6 U}{5} & 0 & 3 U & -\frac{9 U}{5} \\
0 & 0 & -\frac{2 U}{3} & -\frac{4 U}{3} & -2 T+4 U
\end{array}\right) .
$$

From Eqs. (83), (108) and (109), we see that the anomalous dimension matrix is now diagonal in its $T$ dependence.

We have computed the eigenvalues of the matrix above, to check that they are the same as in Eq. (95)) (we will have one fewer, due to the dimensional reduction of our matrix), finding, indeed,

$$
\begin{aligned}
& \lambda_{4}=\lambda_{1}=3 \frac{\alpha_{s}}{\pi} T, \\
& \lambda_{5}=\lambda_{2}=3 \frac{\alpha_{s}}{\pi} U, \\
& \lambda_{6}=\lambda_{3}=3 \frac{\alpha_{s}}{\pi}(T+U), \\
& \lambda_{7}=2 \frac{\alpha_{s}}{\pi}\left[T+U-2 \sqrt{T^{2}-T U+U^{2}}\right], \\
& \lambda_{8}=2 \frac{\alpha_{s}}{\pi}\left[T+U+2 \sqrt{T^{2}-T U+U^{2}}\right] .
\end{aligned}
$$


The eigenvectors have the general form

$$
e_{i}=\left(\begin{array}{c}
e_{i}^{(3)} \\
0^{(5)}
\end{array}\right), i=1,2,3, \quad e_{i}=\left(\begin{array}{c}
0^{(3)} \\
e_{i}^{(5)}
\end{array}\right), i=4 \ldots 8,
$$

where the $e_{i}^{(3)}$ are given explicitly in Eq. (89); $e_{4}^{(5)}, e_{5}^{(5)}$ and $e_{6}^{(5)}$ are given by

$$
e_{4}^{(5)}=\left(\begin{array}{c}
-15 \\
6-\frac{15}{2} \frac{T}{U} \\
-\frac{15}{2} \frac{T}{U} \\
3 \\
1
\end{array}\right), \quad e_{5}^{(5)}=\left(\begin{array}{c}
0 \\
-\frac{3}{2} \\
0 \\
\frac{3}{4}-\frac{3}{2} \frac{T}{U} \\
1
\end{array}\right), \quad e_{6}^{(5)}=\left(\begin{array}{c}
-15 \\
-\frac{3}{2}+\frac{15}{2} \frac{T}{U} \\
\frac{15}{2}-\frac{15}{2} \frac{T}{U} \\
-3 \\
1
\end{array}\right) ;
$$

$e_{7}^{(5)}$ and $e_{8}^{(5)}$ have a rather complicated form, which can be simplified by the reparameterization,

$$
e_{i}^{(5)}=\left(\begin{array}{c}
b_{1}\left(\lambda_{i}^{\prime}\right) \\
b_{2}\left(\lambda_{i}^{\prime}\right) \\
b_{3}\left(\lambda_{i}^{\prime}\right) \\
b_{4}\left(\lambda_{i}^{\prime}\right) \\
1
\end{array}\right), i=7,8
$$

where $\lambda_{i}^{\prime}$ was defined in Eq. (92), and where the $b_{i}$ 's are given by

$$
\begin{aligned}
b_{1}\left(\lambda_{i}^{\prime}\right)= & \frac{3}{U^{2} K^{\prime}}\left[80 T^{4}+103 U^{4}-280 U T^{3}-300 T U^{3}+404 T^{2} U^{2}\right. \\
& \left.+\left(40 T^{3}-16 U^{3}-60 T^{2} U+52 T U^{2}\right) \lambda_{i}^{\prime}\right] \\
b_{2}\left(\lambda_{i}^{\prime}\right)= & \frac{3}{2 K^{\prime}}\left[20 T^{2}-50 U T+44 U^{2}+(10 T-5 U) \lambda_{i}^{\prime}\right] \\
b_{3}\left(\lambda_{i}^{\prime}\right)= & -\frac{3}{2 U K^{\prime}}\left[40 T^{3}-64 U^{3}-120 T^{2} U+130 T U^{2}\right. \\
& \left.\quad+\left(20 T^{2}+13 U^{2}-20 T U\right) \lambda_{i}^{\prime}\right] \\
b_{4}\left(\lambda_{i}^{\prime}\right)= & \frac{3 U}{K^{\prime}}\left(2 T+5 U-2 \lambda_{i}^{\prime}\right)
\end{aligned}
$$

$K^{\prime}$ being the auxiliary function

$$
K^{\prime}=20 T^{2}-20 U T+21 U^{2} .
$$

As $T \rightarrow-\infty$, the eigenvectors $e_{i}$ approach simple linear combinations of the color tensors of Eq. (107). In particular, $e_{7}$ becomes proportional to the color singlet vector $(0,0,0,1,0,0,0,0)$ in Eq. (107), with an eigenvalue that decreases as 6T. This is the most negative eigenvalue, so that, once again, color singlet exchange dominates in the product of hard and soft functions, Eq. (42), for gluon-gluon scattering. Of the remaining eigenvalues, the color structures of the first three are simple products of 
the $f$ and $d$ tensors for all angles (see Eq. (101)). Next, from Eq. (112), we see that $e_{4}$ and $e_{6}$ become degenerate and approach linear combinations of symmetric and antisymmetric octet projectors, while $e_{5}$ corresponds to $10 \oplus \overline{10}$ exchange in the forward limit. Finally, $e_{8}$ approaches pure 27 exchange in the forward limit, with the largest, and hence most suppressed, eigenvalue, $-2 T$. In fact, suppression increases with the dimension of the color representation exchanged. This suggests that radiation by accelerated charges in a nonabelian gauge theory increases with the dimension of the color exchange, not only from singlet to octet, but also to higher representations.

\section{Conclusions}

The anomalous dimension matrix for soft radiation in gluon-gluon scattering completes the set of one-loop calculations that we set out to analyze. An interesting regularity of our results, relevant to near-forward scattering, is that for each flavor combination, logarithms of $t / s$ appear only in diagonal matrix elements in those bases that describe definite color exchange in the $t$-channel. This had been shown previously for the cases of quark-quark and quark-antiquark scattering at one loop [8, 9] and two loops $[10]$. One application of the anomalous dimension matrices and their eigenvalues is for the resummation of threshold singularities to next-to-leading logarithm in jet cross sections 21] at fixed rapidities. This was the original motivation for our study. We are hopeful, however, that the formalism of resumming coherent noncollinear soft gluon radiation in terms of color mixing will have a variety of other uses as well.

\section{Acknowledgements}

This work was supported in part by the National Science Foundation, under grant PHY9309888 and by the PPARC under grant GR/K54601. We wish to thank Eric Laenen, Jack Smith, and Ramona Vogt for many helpful conversations.

\section{A Feynman rules for eikonal diagrams in axial gauge}

In this Appendix we summarize the Feynman rules we have used in the evaluation of the eikonal diagrams of Fig. 17. We refer to Fig. 7.

The propagator for a quark, antiquark or gluon eikonal line (Fig. 7) is given by

$$
\frac{i}{\delta v \cdot q+i \epsilon}
$$

where $\delta=+1(-1)$ for the momentum $q$ flowing in the same (opposite) direction as the dimensionless vector $v$. The interaction vertex for a quark or antiquark eikonal line (Fig. [la, b) is

$$
-i g_{s} T_{F b a}^{c} \Delta v^{\mu}
$$


with $\Delta=+1(-1)$ for a quark (antiquark).

For the gluon eikonal vertex (Fig. $7 \mathrm{c}$ ), we may take

$$
-g_{s} f^{a b c} \Delta v_{\mu}
$$

where we agree to read the color indexes $a, b, c$ anticlockwise, and where $\Delta=+1(-1)$ for the gluon located below (above) the eikonal line.

Finally in our calculations we employ the general axial gauge gluon propagator,

$$
D^{\mu \nu}(k)=\frac{-i}{k^{2}+i \epsilon} N^{\mu \nu}(k), \quad N^{\mu \nu}(k)=g^{\mu \nu}-\frac{n^{\mu} k^{\nu}+k^{\mu} n^{\nu}}{n \cdot k}+n^{2} \frac{k^{\mu} k^{\nu}}{(n \cdot k)^{2}},
$$

with $n^{\mu}$ the axial gauge-fixing vector.

\section{B Evaluation of one-loop eikonal vertex correc- tions}

We refer to Fig. 国, where we have represented the UV divergent $O\left(\alpha_{s}\right)$ contributions to the eikonal vertex $W_{I}^{(\mathrm{f})}$. The counterterms for $W_{I}^{(\mathrm{f})}$ are ultraviolet divergent coefficients, depending on the external momenta and on the axial gauge fixing vector, times our basis color tensors, $c_{I}^{(\mathrm{f})}$.

At Born level the eikonal vertices describe only color flow, and the relation

$$
W_{I, \text { Born }}^{(\mathrm{f})}=c_{I}^{(\mathrm{f})}
$$

holds.

The one-loop corrections can be written in matrix notation as

$$
W_{1 \text { loop }}^{(\mathrm{f}), T}=c^{(\mathrm{f}), T} Z_{S}=W_{\text {Born }}^{(\mathrm{f}), T} Z_{S},
$$

where the superscript $T$ indicates transpose. The dimension of the vectors (and therefore the rank of the matrix of renormalization constants) depends on the specific partonic process, as discussed in detail above.

We denote the kinematic part of the one-loop vertex correction to $W_{I}^{(\mathrm{f})}$, with the virtual gluon linking lines $v_{i}$ and $v_{j}$, as $\omega_{i j}\left(\delta_{i} v_{i}, \delta_{j} v_{j}, \Delta_{i}, \Delta_{j}\right)$, with $\delta_{i}$ 's and $\Delta_{i}$ 's defined in Appendix A. Its expression is

$$
\begin{aligned}
\omega_{i j}\left(\delta_{i} v_{i}, \delta_{j} v_{j}, \Delta_{i}, \Delta_{j}\right)= & g_{s}^{2} \int \frac{d^{n} q}{(2 \pi)^{n}} \frac{-i}{q^{2}+i \epsilon}\left\{\frac{\Delta_{i} \Delta_{j} v_{i} \cdot v_{j}}{\left(\delta_{i} v_{i} \cdot q+i \epsilon\right)\left(\delta_{j} v_{j} \cdot q+i \epsilon\right)}\right. \\
& \left.-\frac{\Delta_{i} v_{i} \cdot n}{\left(\delta_{i} v_{i} \cdot q+i \epsilon\right)} \frac{P}{(n \cdot q)}-\frac{\Delta_{j} v_{j} \cdot n}{\left(\delta_{j} v_{j} \cdot q+i \epsilon\right)} \frac{P}{(n \cdot q)}+n^{2} \frac{P}{(n \cdot q)^{2}}\right\},
\end{aligned}
$$

where $P$ stands for principal value,

$$
\frac{P}{(q \cdot n)^{\beta}}=\frac{1}{2}\left(\frac{1}{(q \cdot n+i \epsilon)^{\beta}}+(-1)^{\beta} \frac{1}{(-q \cdot n+i \epsilon)^{\beta}}\right) .
$$


We rewrite (122) as

$$
\begin{aligned}
\omega_{i j}\left(\delta_{i} v_{i}, \delta_{j} v_{j}, \Delta_{i}, \Delta_{j}\right)= & \mathcal{S}_{i j}\left[I_{1}\left(\delta_{i} v_{i}, \delta_{j} v_{j}\right)-\frac{1}{2} I_{2}\left(\delta_{i} v_{i}, n\right)-\frac{1}{2} I_{2}\left(\delta_{i} v_{i},-n\right)\right. \\
& \left.-\frac{1}{2} I_{3}\left(\delta_{j} v_{j}, n\right)-\frac{1}{2} I_{3}\left(\delta_{j} v_{j},-n\right)+I_{4}\left(n^{2}\right)\right]
\end{aligned}
$$

where $\mathcal{S}_{i j}$ is an overall sign

$$
\mathcal{S}_{i j}=\Delta_{i} \Delta_{j} \delta_{i} \delta_{j}
$$

The ultraviolet poles of the integrals are [7, 8

$$
\begin{aligned}
I_{1}^{U V ~ p o l e ~} & =\frac{\alpha_{s}}{\pi}\left\{\frac{2}{\epsilon^{2}}-\frac{1}{\epsilon}\left[\gamma+\ln \left(\delta_{i} \delta_{j} \frac{v_{i} \cdot v_{j}}{2}\right)-\ln (4 \pi)\right]\right\}, \\
I_{2}^{U V ~ p o l e ~} & =\frac{\alpha_{s}}{2 \pi}\left\{\frac{2}{\epsilon^{2}}-\frac{1}{\epsilon}\left[\gamma+\ln \left(\nu_{i}\right)-\ln (4 \pi)\right]\right\}, \\
I_{3}^{U V ~ p o l e ~} & =\frac{\alpha_{s}}{2 \pi}\left\{\frac{2}{\epsilon^{2}}-\frac{1}{\epsilon}\left[\gamma+\ln \left(\nu_{j}\right)-\ln (4 \pi)\right]\right\}, \\
I_{4}^{U V ~ p o l e ~} & =-\frac{\alpha_{s}}{\pi} \frac{1}{\epsilon},
\end{aligned}
$$

where all the gauge dependence is through the variable

$$
\nu_{i}=\frac{\left(v_{i} \cdot n\right)^{2}}{|n|^{2}} .
$$

The double poles cancel in (124), giving

$$
\omega_{i j}\left(\delta_{i} v_{i}, \delta_{j} v_{j}, \Delta_{i}, \Delta_{j}\right)=-\mathcal{S}_{i j} \frac{\alpha_{s}}{\pi \epsilon}\left[\ln \left(\frac{\delta_{i} \delta_{j} v_{i} \cdot v_{j}}{2}\right)-\frac{1}{2} \ln \left(\nu_{i} \nu_{j}\right)+1\right] .
$$

In order to obtain contributions to the different entries of the matrix of renormalization constants, the above expression has still to be multiplied by the color decomposition of its corresponding diagram into the basis color structures [7, [17, 18].

\section{References}

[1] G.T. Bodwin, Phys. Rev. D31, 2616 (1985); E. D34, 3932 (1986); J.C. Collins, D.E. Soper and G. Sterman, Nucl. Phys. B261, 104 (1985).

[2] J.C. Collins, D.E. Soper and G. Sterman, Nucl. Phys. B308, 833 (1988).

[3] J.C. Collins, D.E. Soper and G. Sterman, in Perturbative Quantum Chromodynamics, ed. A. H. Mueller (World Scientific, Singapore, 1989), p. 1.

[4] J.C. Collins and D.E. Soper, Nucl. Phys. B193, 381 (1981).

[5] Y.L. Dokshitzer, D.I. Dyakonov, and S.I. Troyan, Phys. Lett. 79B, 269 (1978);

G. Parisi and R. Petronzio, Nucl. Phys. B154, 427 (1979). 
[6] J.C. Collins, D.E. Soper, and G. Sterman, Nucl. Phys. B250, 199 (1985); C.T.H. Davies and W.J. Stirling, Nucl. Phys. B244, 337 (1984); C.T.H. Davies, B.R. Webber, and W.J. Stirling, Nucl. Phys. B256, 413 (1985); P.B. Arnold and R.P. Kauffman, Nucl. Phys. B349, 381 (1991); G. Ladinsky and C.-P. Yuan, Phys. Rev. D 50, 4239 (1994).

[7] J. Botts and G. Sterman, Nucl. Phys. B325, 62 (1989).

[8] M.G. Sotiropoulos and G. Sterman, Nucl. Phys. B419, 59 (1994).

[9] G.P. Korchemsky, Phys. Lett. B 325, 459 (1994).

[10] I.A. Korchemskaya and G. P. Korchemsky, Nucl. Phys. B437, 127 (1995).

[11] Y.L. Dokshitzer, D.I. Dyakonov and S.I. Troyan, Phys. Rep. 58, 269 (1980); A.H. Mueller, Phys. Lett. 104B, 161 (1981); A. Bassetto, M. Ciafaloni, G. Marchesini and A.H. Mueller, Nucl. Phys. B207, 189 (1982); Y.L. Dokshitzer, V.A. Khoze, S.I. Troyan and A.H. Mueller, Rev. Mod. Phys. 60, 373 (1988); G. Marchesini and B.R. Webber, Nucl. Phys. B310, 461 (1988); Y.L. Dokshitzer, V.A. Khoze and S.I. Troyan, in Perturbative Quantum Chromodynamics, ed. A.H. Mueller (World Scientific, Singapore, 1989), p. 241; Y.L. Dokshitzer, V.A. Khoze, G. Marchesini and B.R. Webber, Phys. Lett. B245, 243 (1990).

[12] G. Sterman, Nucl. Phys. B281, 310 (1987).

[13] S. Catani and L. Trentadue, Nucl. Phys. B327, 323 (1989); B353, 183 (1991); L. Magnea, Nucl. Phys. B349, 703 (1991).

[14] E. Laenen, J. Smith, and W.L. van Neerven, Nucl. Phys. B369, 543 (1992); Phys. Lett. B321, 254 (1994); N. Kidonakis and J. Smith, Phys. Rev. D51, 6092 (1995); Mod. Phys. Lett. A11, 587 (1996).

[15] E.L. Berger and H. Contopanagos, Phys. Lett. B361, 115 (1995); Phys. Rev. D54, 3085 (1996); ibid. D57, 253 (1998).

[16] S. Catani, M.L. Mangano, P. Nason, and L. Trentadue, Phys. Lett. B378, 329 (1996); Nucl. Phys. B478, 273 (1996).

[17] N. Kidonakis and G. Sterman, Phys. Lett. B387, 867 (1996); Nucl. Phys. B505, 321 (1997); in proceedings of Deep Inelastic Scattering and QCD, 5th International Workshop, ed. J. Repond and D. Krakauer (AIP Conf. Proc. No. 407, American Institute of Physics, Woodbury, NY, 1997), p. 1035, hep-ph/9708353.

[18] N. Kidonakis, SUNY at Stony Brook Ph.D. Thesis (1996), hep-ph/9606474.

[19] N. Kidonakis, J. Smith and R. Vogt, Phys. Rev. D56, 1553 (1997); N. Kidonakis, contribution presented at the QCD 97 Euroconference, Montpellier, July 3-9, 1997, hep-ph/9708439; N. Kidonakis and R. Vogt, in preparation. 
[20] R. Bonciani, S. Catani, M.L. Mangano and P. Nason, hep-ph/9801375.

[21] N. Kidonakis, G. Oderda and G. Sterman, hep-ph/9801268.

[22] Y.L. Dokshitzer, V.A. Khoze and S.I. Troyan, in "Physics in Collision VI", Proceedings of the International Conference, Chicago, Illinois, 1986, ed. M. Derrick (World Scientific, Singapore, 1987), p. 417; J.D. Bjorken, Phys. Rev. D47, 101 (1993); A.D. Martin, M.G. Ryskin and V.A. Khoze, Phys. Rev. D56, 5867, (1997); O.J.P. Eboli, E.M. Gregores and F. Halzen, hep-ph/9708283; R. Oeckl, D. Zeppenfeld, hep-ph/9801257.

[23] H. Contopanagos, E. Laenen and G. Sterman, Nucl. Phys. B484, 303 (1997).

[24] G.P. Korchemsky and G. Marchesini, Phys. Lett. B313, 433 (1993).

[25] R.A. Brandt, F. Neri, and M.-a. Sato, Phys. Rev. D24, 879 (1981).

[26] A.M. Polyakov, Nucl. Phys. B164, 171 (1979); I.Ya. Aref'eva, Phys. Lett. 93B, 347 (1980); V.S. Dotsenko and S.N. Vergeles, Nucl. Phys. B169, 527 (1980).

[27] S.V. Ivanov and G.P. Korchemsky, Phys. Lett. B154, 197 (1985); G.P. Korchemsky and A.V. Radyushkin, Phys. Lett. B171, 459 (1986); Nucl. Phys. B283, 342 (1987); G.P. Korchemsky, Phys. Lett. B217, 330 (1989); ibid. B220, 629 (1989).

[28] A.J. Macfarlane, A. Sudbery and P.H. Weisz, Commun. Math. Phys. 11, 77 (1968).

[29] L. Dixon in QCD and Beyond, Proceedings of the Theoretical Advanced Study Institute in Elementary Particle Physics (TASI-95), ed. D.E. Soper (World Scientific, Singapore, 1996), p. 539, hep-ph/9601359.

[30] J. Bartels, Z. Phys. C60, 471 (1993). 


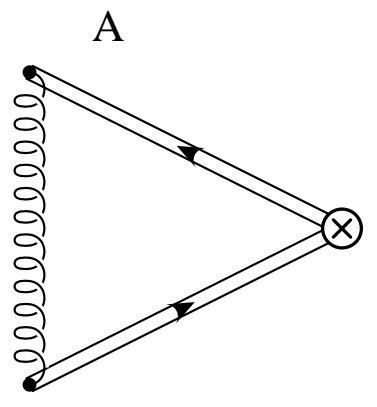

B

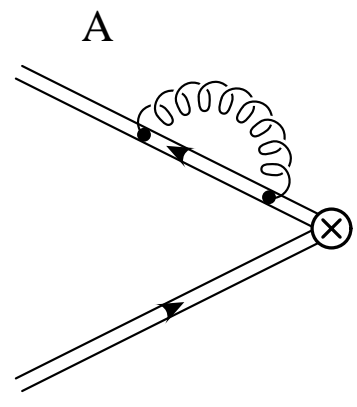

$\mathrm{B}$

Figure 1: Eikonal vertex correction and eikonal self-energy for the Drell Yan process.
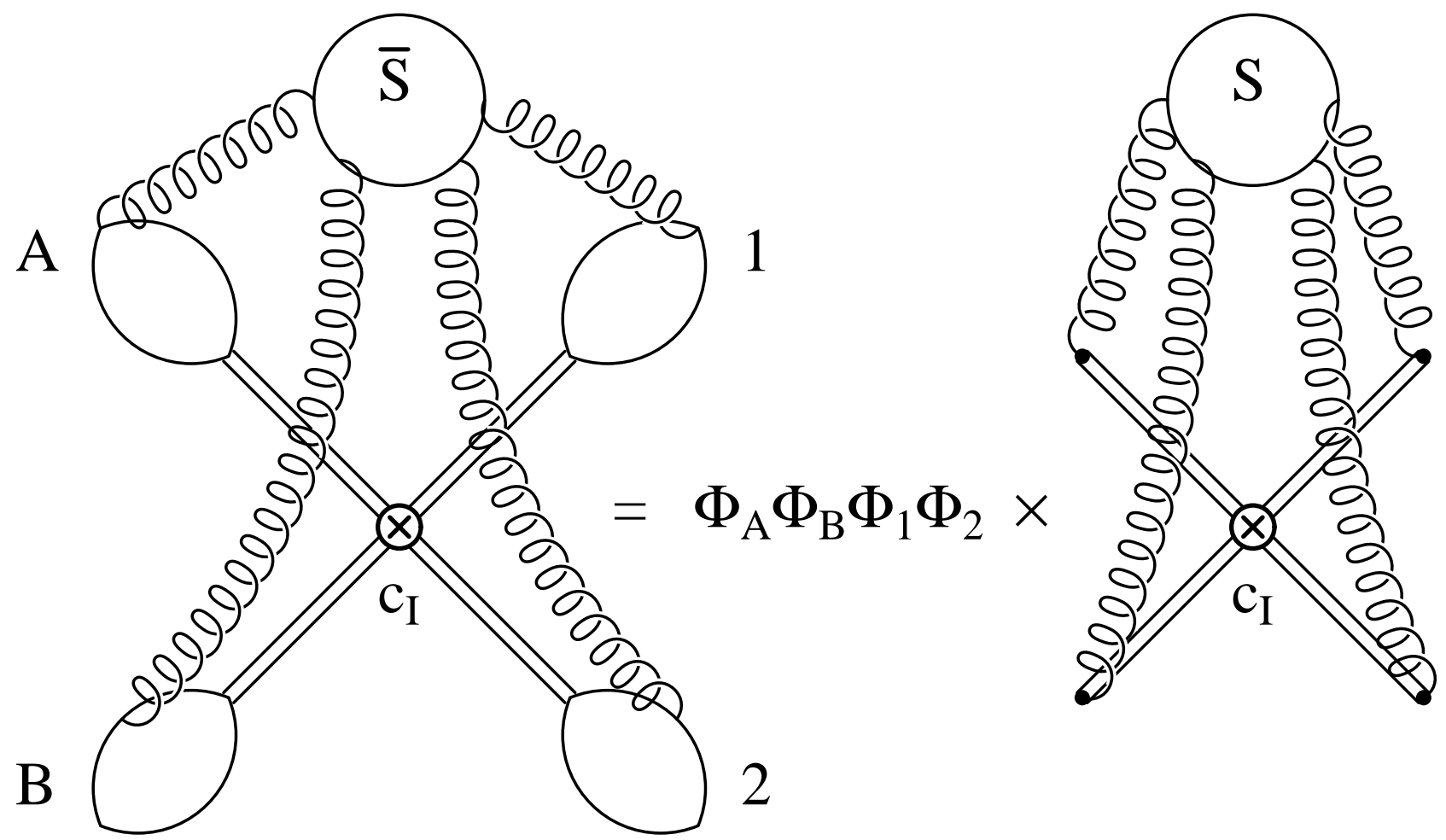

Figure 2: Identity representing factorization in leading regions of corrections to the eikonal vertex $c_{I} . \bar{S}$ represents lines of zero momentum, while A, B, 1 and 2 label jet subdiagrams. 


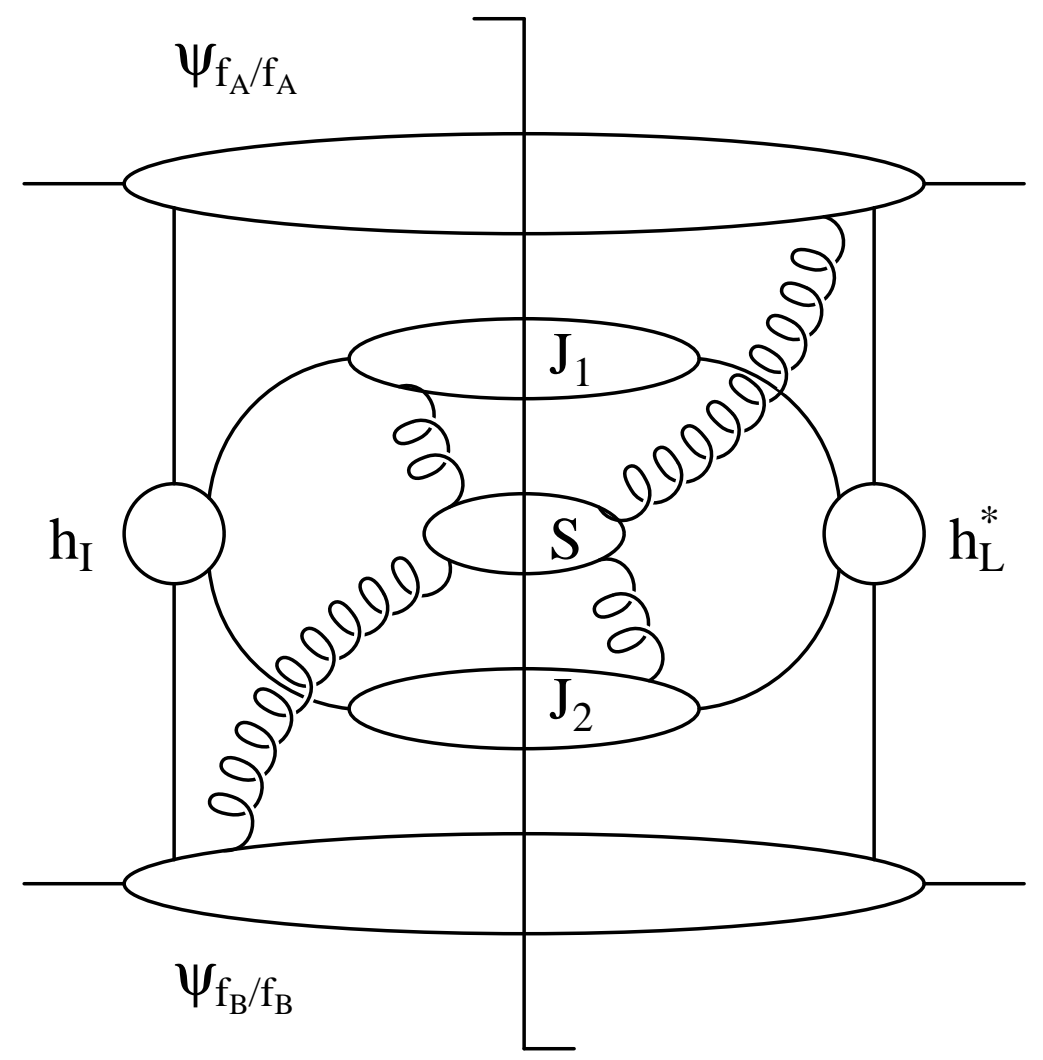

Figure 3: Reduced diagram representing the leading region for the dijet cross section. 

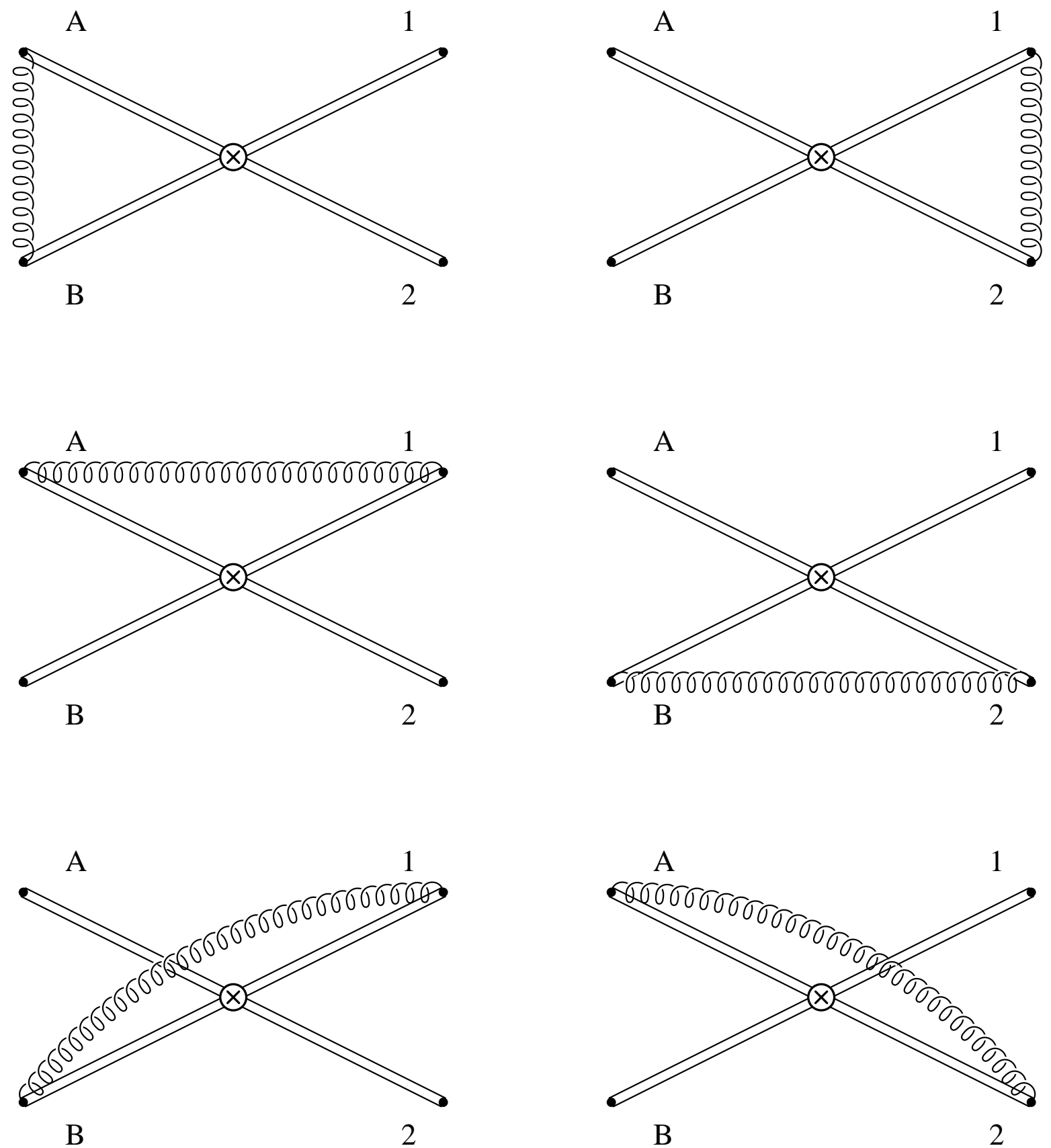

Figure 4: Eikonal vertex corrections for partonic processes contributing to the soft anomalous dimension matrices. 

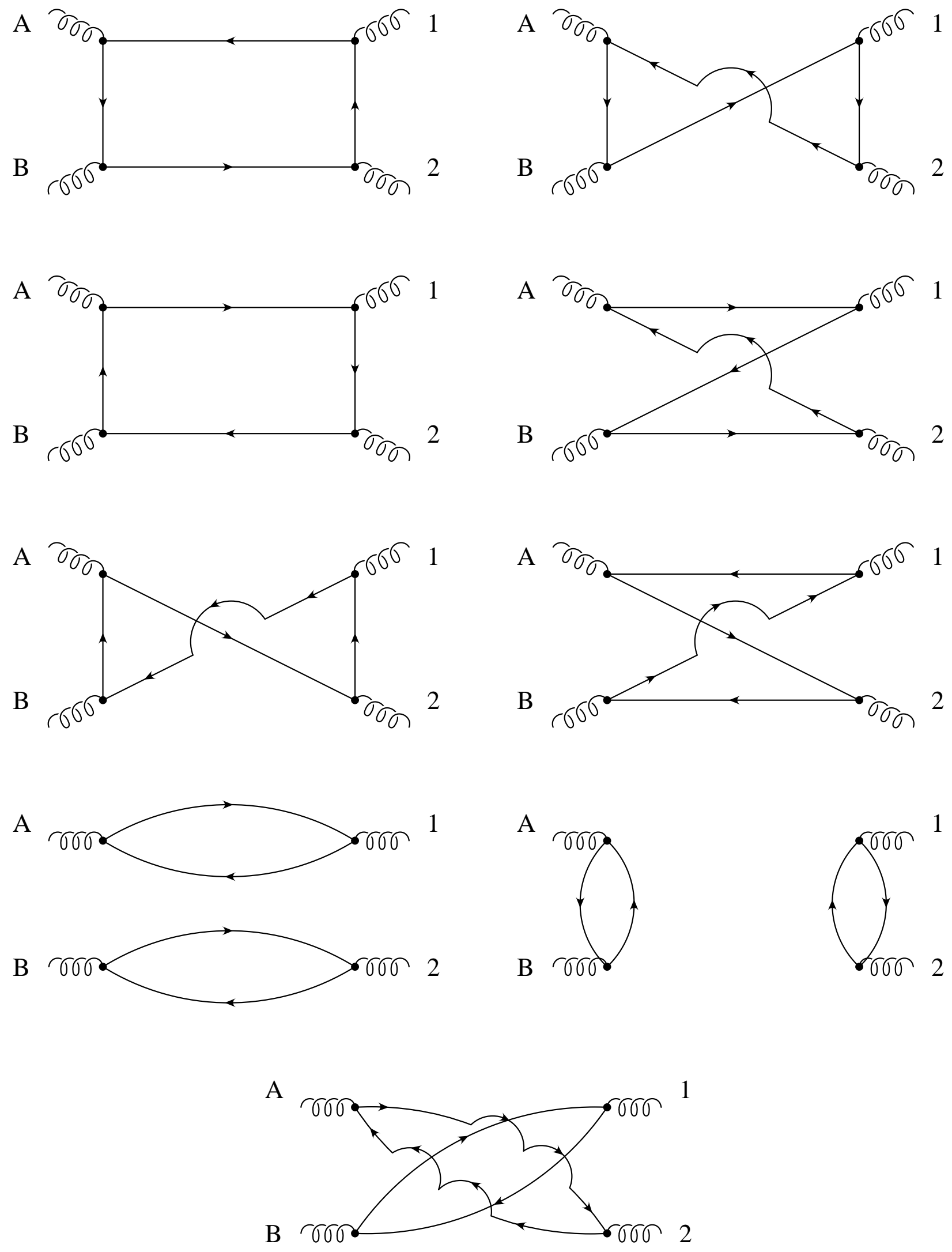

Figure 5: Graphical representation of the initial color basis for the $g g \rightarrow g g$ process. All lines have color content only. 
r के
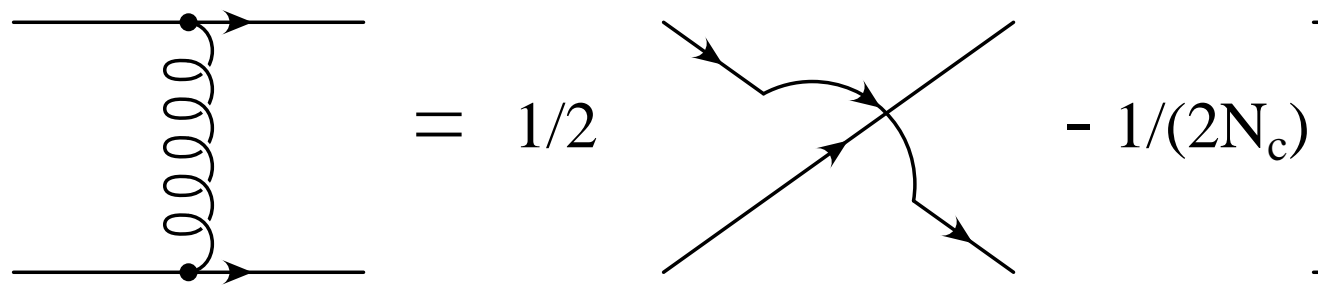

Figure 6: Graphical representation for the color decompositions in Eq. (79). 


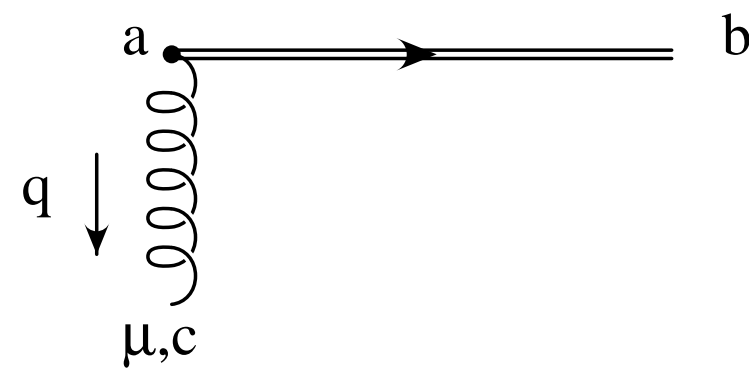

$=g_{s}\left(T_{F}^{c}\right)_{b a} v^{\mu}(-v \cdot q)^{-1}$

(a)

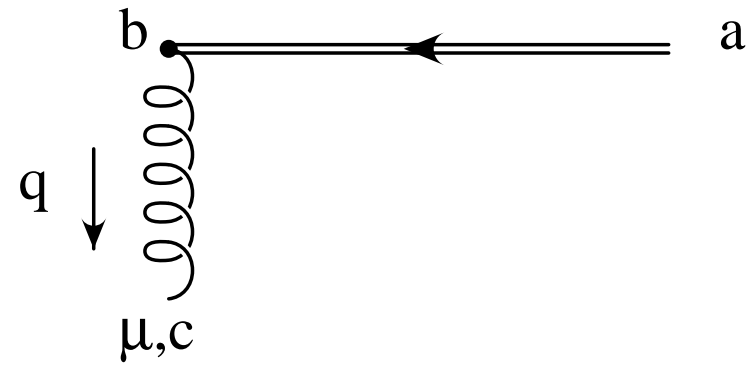

$=-g_{s}\left(T_{F}^{c}\right)_{b a} v^{\mu}(-v \cdot q)^{-1}$

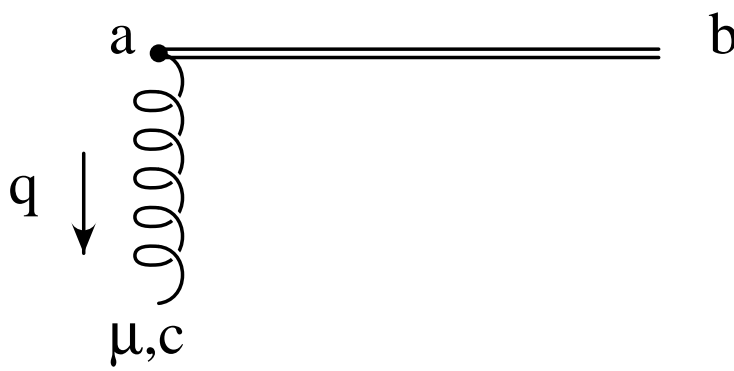

$=-i g_{s} f^{b a c} v^{\mu}(-v \cdot q)^{-1}$

(c)

Figure 7: Illustration of the eikonal Feynman rules for a quark (a), antiquark (b) and gluon (c) eikonal line. 